\title{
Effectiveness of Routine Physical Therapy with and Without Pain Release Phenomenon in Patello- Femoral Pain Syndrome
}

\author{
Sana Shahid ${ }^{1}$, Dr. Ashfaq Ahmed ${ }^{2}$, Dr. Umair Ahmed ${ }^{3}$ \\ ${ }^{1}$ Scholar of Master of Philosophy in Physical Therapy (Musculoskeletal), University Institute of Physical Therapy (UIPT), Faculty of Allied \\ Health Sciences, The University o Lahore \\ ${ }^{2}$ Supervisor, BSPT, DPT (KEMU), M. Phil,PT (KEMU), University Institute of Physical Therapy (UIPT), Faculty of Allied Health \\ Sciences, The University o Lahore \\ ${ }^{3}$ Co-Supervisors, BSPT(PAK), MSC. NEUROLOGY (UK), University Institute of Physical Therapy (UIPT), Faculty of Allied Health
} Sciences, The University o Lahore

\begin{abstract}
Background: Physical therapy intervention is the mainstay for treating patellofemoral pain syndrome i.e. Retropatellar pain. Despite of the availability of a number of treatment options there is lack of agreement on any specific treatment approach of Patellofemoral pain syndrome. Objective: The objective of this study was to compare the effectiveness of physical therapy treatment with and without Pain Release Phenomenon in patellofemoral pain syndrome. Materials and methods: It was a double blind, randomized controlled trial. 60 participants were randomly assigned to two groups. The treatment was provided for six weeks thrice every week. Group A received conventional/standardized Physical therapy treatment and Group B received conventional/standardized Physical therapy treatment along with pain release phenomenon. The baseline measurements were taken at the beginning and at six weeks posttrial. Results: Patients did not differ in baseline pain and disability level in both groups. Six weeks post intervention group B receiving the physical therapy with pain relief phenomenon showed markedly improved functional status ( $p$-value=0.01, Confidence interval $=95 \%$ ) and reduction in level of pain (p-value $=0.02$, Confidence interval=95\%) as compared to group A. Effect sizes for both VAS and Functional index questionnaire were large. Conclusions: This study concludes that Pain release phenomenon is an effective technique in reducing pain and improving function of knee in patients with patellofemoral pain when combined with conventional treatment and home exercise plan over a period of six weeks.
\end{abstract}

Keywords: Functional Status, Knee Pain, Patellofemoral Pain, Physical Therapy, Visual Analogue Scale

Alternative Hypothesis

Physical Therapy with Pain Release Phenomenon is more effective than Physical Therapy without Pain Release Phenomenon, in relieving pain in Patello-Femoral Pain Syndrome [PFPS].

\section{Introduction}

There is no clear consensus in the literature on the terminology for pain in the anterior part of theKnee. Patellofemoral pain syndrome (Patellofemoral pain syndrome) is difficult to define, as patients experience a variety of symptoms from the patellofemoral joint with different levels of pain and physical impairment. The terminology is thus still widely discussed. Anterior knee pain, chondromalacia patella, patellofemoral arthralgia, patellar pain, patellar pain syndrome and patellofemoral pain are often used synonymously with Patellofemoral pain syndrome. $^{(1,2)}$

The patellofemoral joint comprises the patella and the femoral trochlea. The patella acts as a lever and also increases the moment arm of the patellofemoral joint, the quadriceps and patellar tendons.8 Contact of the patella with the femur is initiated at 20 degrees of flexion and increases with further knee flexion, reaching a maximum at 90 degrees. ${ }^{(3)}$

To assess the degree of PFPS, a variety of symptoms and different levels of pain and physical impairment must be considered. The etiology is still unclear in many patients.
Three major contributing factors increasing the risk of developing PFPS are discussed: malalignment of the lower extremity and/or the patella, muscular imbalance of the lower extremity, and over activity. ${ }^{(4)}$ As patellofemoral pain syndrome is the most common cause of anterior knee pain in the outpatient, a variety of treatments for patellofemoral pain syndrome are implemented. However, there is little supporting evidence. Most patients with patellofemoral pain syndrome respond well to conservative therapy. ${ }^{(5)}$

\section{Pain Release Phenomenon}

The Pain Release Phenomenon Techniques (PRPS) is a manual therapy technique introduced by Brian Mulligan for the chronic pain management in the extremities. In this technique, joint compression, muscular contraction or stretch is used as the pain provoking stimuli and the stimuli is maintained for 15- 20 seconds. If indicated, the pain will reduce in this period ${ }^{(6)}$

The primary aim of the study is to find the effectiveness of physical therapy treatment with and without Pain Release Phenomenon. It is one of the new techniques and one of the important advantage of the rational treatment is that further 


\section{International Journal of Science and Research (IJSR) \\ ISSN (Online): 2319-7064 \\ Index Copernicus Value (2013): 6.14 | Impact Factor (2015): 6.391}

invasive procedure like surgery can be avoided. Wastage of time, energy and money of the patient is avoided.

\section{Literature Review}

Basic knowledge is lacking and no strong scientific evidence has been presented in the literature on the nature and etiology of Patellofemoral pain syndrome. This could explain why there are so many treatment protocols described in the literature. Different treatment protocols are being used mainly depending upon clinical guidelines of different clinical facilities. ${ }^{(7-11)}$

Physical interventions are the mainstay of treatment for Patellofemoral pain syndrome (Patellofemoral pain syndrome). Physiotherapy is the most common of all physical interventions and includes specific vastusmedialisobliquus or general quadriceps strengthening and/or realignment procedures (tape, brace, stretching). ${ }^{(12)}$ These treatments appear to be based on sound theoretical rationale and have attained widespread acceptance, but evidence for the efficacy of these interventions is not well established. This study was a trial to investigate not only the available evidence for physical interventions for Patellofemoral pain syndrome, and also to see the effective approach. $^{(13,14)}$

Fukuda et al ,2010 conducted a randomized controlled trial for the treatment of PFPS, in this study hip strengthening exercises and knee strengthening exercises were done. Combined effect of both exercises was found to be effective during a treatment session of 4 weeks. ${ }^{(7)}$ In a study conducted by Ferber et al in 2011 a 3 weeks treatment containing hipabductor muscle-strengthening was effective in increasing muscle strength and decreasing pain in individuals with PFPS. ${ }^{(4)}$ In 2011 Bolgla et al conducted a systematic review of the literature from 2000 to 2010 for the conservative management of patellofemoral pain syndrome. Evidence supported the continued use of quadriceps exercise for the conservative management of PFPS. However, inconsistent or limited data was found regarding the other interventions. Study stated that ongoing investigations are needed to better understand its effect on PFPS. ${ }^{(8)}$

\section{Aims and Objectives}

1) To find the effectiveness of physical therapy treatment with and without Pain Release Phenomenon.

2) To correlate between pain and ROM.

\section{Operational Definitions}

\section{Physical therapy without pain relieve phenomenon}

This group was provided with traditional physical therapy intervention for Patellofemoral pain syndrome. This will consist of

\section{Restoring Flexibility of Restricted Tissue}

Any structures that can be contributing to faulty mechanics was identified and was established a stretching program. The gastrocnemius, soleus, quadriceps, and hamstring muscles have been identified as specific muscles with decreased flexibility in individuals with patellofemoral dysfunction.

\section{Patellar Mobilization}

Patellar mobilization-medial glide. patientwas positioned in side-lying, by stabilizing the femoral condyles with one hand under the femur and the patella was glided medially with the base of the other hand.

\section{Medial Tipping of the Patella}

The patient was positioned in supine. The thenar eminence was placed at the base of the hand over the medial aspect of the patella. Direct posterior force will tip the patella medially. While the patella was held in this position, friction massage would also be applied with the other hand along the lateral border.

\section{Quadriceps Setting (Quad Sets)}

This was done in pain-free positions. The patient was placed to set the quads with the knee in various positions while focusing on tension development in the VMO.

\section{Quad Sets with Straight-Leg Raising.}

Because many fibers of the VMO originate on the adductor tendons and medial intramuscular septum, some popular exercise programs suggest that by laterally rotating the femur while performing. SLR exercises the adductors contract and provide a firm base for the VMO. This was included in interventional plan.

\section{Short-Arc Terminal Extension.}

It was performed by beginning with the patient supine and knee flexed around 20 degree. If tolerated and the motion is not painful, light resistance was added at the ankle. Strengthening in terminal extension trains the muscle to function where it is least efficient because of its shortened position and where there was minimal patellar compression because it was superior to the femoral groove.

\section{Physical therapy with pain relieve phenomenon}

In addition to aforementioned treatment, the Pain Release Phenomenon was applied to patients of group B. This is a procedure as described by Brian Mulligan.

\section{Pain Release Phenomenon}

The technique which provokes pain was selected; and the pain provoked should settle down within 15-20 seconds (for smaller joints) and 25-30 seconds (for larger joints) $\rightarrow$ The range at which "THE" pain starts was evaluated $\rightarrow$ Therapist will maintain pressure for 15 - 20 seconds $\rightarrow$ If pain reduces within 15 -20 seconds, it was started with new PRP in new available range with increased force $\rightarrow$ If pain doesn't reduce within 15-20 seconds ,it implies that pressure being applied is too high. Hence the pressure should be reduced to a level so that provoked pain gets reduced within 20 seconds $\rightarrow$ If pain reduces before 10 seconds, it implies that pressure being applied is too low. Hence the pressure should be increased to a level so that provoked pain gets reduced within 15 - 20 seconds and not before 10 seconds $\rightarrow$ In addition to applied pressure, Physiological movement or accessory movement may be added along with the selected PRPs $\rightarrow$ Continue to perform PRPs until a substantial amount of pain relief is achieved during a session. 


\section{Materials and Methods}

\section{Study Design:}

Study wasrandomized controlled trial.

\section{Setting}

Data was collected from University Physical Therapy Clinic, The University of Lahore.

\section{Sample Size:}

Total population of 80 both males and females below 40 years of age are selected for the study. Amongpopulation of 80, 40 are selected for conventional therapy and 40 for New therapy (Pain Release Phenomenon).

\section{Duration of Study}

It was completed within 6 months after the approval of synopsis.

\section{Sample Selection Criteria}

\section{Inclusion Criteria}

Patients with following characteristics was included;

- Patients with age less than 40 years

- anterior knee pain or retro-patellar pain

- Patellofemoral pain syndrome insidious onset for at least one month reported on at least two of the following: prolonged sitting, ascending or descending stairs, squatting, running, kneeling and hopping/jumping

\section{Exclusion Criteria}

Patients with following conditions was excluded,

- Knee surgery within the previous 3 months

- A history of patellar dislocation/subluxation (noninsidious

- Clinical evidence of a current knee condition other than Patellofemoral pain syndrome or were

- Currently taking non-steroidal anti-inflammatory drugs or corticosteroid medication.

\section{Methodology}

The number of patients, sample size extracted on basis on pilot study was distributed equally in following two groups

Group A received physical therapy intervention without Pain Release Phenomenon which includes patellar mobilization, quadriceps strengthening, quads set with straight leg raising and short arc terminal extension.

Group B received physical therapy intervention with Pain Release Phenomenon.the pain provoked should settle down within 15-20 seconds (for smaller joints) and 25-30 seconds (for larger joints) $\rightarrow$ The range at which "THE" pain starts was evaluated $\rightarrow$ Therapist will maintain pressure at P1 for 15- 20 seconds $\rightarrow$ If pain reduces within $15-20$ seconds, it was started with new PRP in new available range with increased force $\rightarrow$ If pain doesn't reduce within 15-20 seconds ,it implies that pressure being applied is too high. Hence the pressure should be reduced to a level so that provoked pain gets reduced within 20 seconds $\rightarrow$ If pain reduces before 10 seconds, it implies that pressure being applied is too low. Hence the pressure should be increased to a level so that provoked pain gets reduced within $15-20$ seconds and not before 10 seconds $\rightarrow$ In addition to applied pressure, Physiological movement or accessory movement may be added along with the selected PRPs $\rightarrow$ Continue to perform PRPs until a substantial amount of pain relief is achieved during a session.

\section{Randomization}

Randomization was performed using computer generated design adaptive allocation. It was used to balance six variable of baselines in all groups. The baseline variables will include pain and disability scores, age, gender, confidence in physical therapy management and pain relief phenomenon and any history of previously taken these interventions

\section{Concealment Of Allocation}

Concealment of allocation to all groups was ensured, from all study personnel and participants by entry of data into computer randomization program immediately. Patient coordinators was called in to research staff in allocating groups, and research staff will enter data into computer program. Patient coordinator will assign patient to group by a sealed envelope as patient identification, and envelop was placed in patient's clinic file.

\section{Blindness}

The clinicians would not be blinded but data assessor and patients was managed to ensure to be blinded about intervention type and group.

\section{Outcome Measures}

Visual Analogue Scale and Functional Index Questionnaire was used to measure progress

\section{Data Analysis}

After taking informed written consent.Data was collected through Patient Performa. Point measures and standard deviation was calculated for demographics and base line variables. Means of two groups was analyzed through Paired Sample t-test. Within group improvement was measured through Wilcoxon Test. Statistical Significance was measured through p-value, taking below 0.05 as significant and more than 0.05 as non-significant.

\section{Results}

During the 6 months duration 102 participants were referred to this trial out of which 60 (59\%) patients fulfilled the eligibility criteria. Sixty patients were randomly allocated into two groups. The details of the two groups are given in table 2.

Five patients could not give follow-up and hence were dropped out of the study.

Participants in group A showed an adherence to exercise for $86 \%$ and those in group B showed adherence for $88 \%$ of the required days.

The comparison of patients in both groups showed no significant differences in mean Age, height, weight and 


\section{International Journal of Science and Research (IJSR) \\ ISSN (Online): 2319-7064 \\ Index Copernicus Value (2013): 6.14 | Impact Factor (2015): 6.391}

Body Mass index (p-value $>0.05$ ).

Chi-square tests for comparisons between two groups showed no significant differences in Sex, dominant leg and side with greater pain ( $p$-value $>0.05$ ) Measured on visual analogue scale (VAS)

Patients did not differ in baseline pain and disability level in both groups. Six weeks post intervention group B receiving the physical therapy with pain relief phenomenon showed markedly improved functional status (p-value $=0.01$, Confidence interval=95\%) and reduction in level of pain (pvalue $=0.02$, Confidence interval $=95 \%$ ) as compared to group A. Effect sizes for both VAS and Functional index questionnaire were large.

\section{List of Tables}

Table 1

\begin{tabular}{l} 
Week 1 to 3 \\
\hline Stretches \\
- Hamstrings (3-5 Repetitions 15 seconds) \\
- Gastro soleus (3-5 Repetitions 15 seconds) \\
- Illiotibial band ( 3 -5 Repetitions 15 seconds) \\
- Rectus femoris (3 -5 Repetitions 15 seconds) \\
Patellar mobilization combined with soft-tissue mobilization to \\
lateral structures \\
Medial tipping of patella \\
Quadriceps setting focussing on VMO (10 repetitions with 10 \\
seconds hold) \\
Short Arc terminal extension (10 repetitions 3 sets) \\
Week 3-6 \\
Stretches continued \\
Patellar tapping \\
Quadriceps and Hip muscle strengthening \\
- Isometric hip abduction while standing (4 sets of 30 seconds \\
hold) \\
- Side step-downs ( 3 sets of 5 repetitions) \\
- Wall squats ( 40 o knee flexion) ( 3 sets of 5 repetitions) \\
- inside leg raise (3 sets of 5 repetitions) \\
- Single leg balance with knee straight and bent (3-5 repetitions 5 \\
seconds hold) \\
- Resisted knee extension with theraband (2 sets of 10 repetitions) \\
\hline Home-exercise plan: a combination of self-stretches and \\
strengthening exercises.
\end{tabular}

Table 2

\begin{tabular}{|c|c|c|c|c|c|}
\hline & \multicolumn{2}{|c|}{ Group A (N=30) } & \multicolumn{2}{c|}{ Group B (N=30) } & \\
\hline & Mean \pm SD & Range & Mean \pm SD & Range & P-value \\
\hline Age (years) & $29 \pm 1.66$ & $19-44$ & $27 \pm 1.66$ & $17-41$ & 0.08 \\
\hline $\begin{array}{c}\text { Height } \\
\text { (meters) }\end{array}$ & $1.68 \pm 0.03$ & $1.50-$ & $1.68 \pm 0.01$ & $1.53-$ & 0.63 \\
\hline Weight $(\mathrm{kgs})$ & $75.6 \pm 1.31$ & $52-105$ & $76.1 \pm 2.31$ & $53-100$ & 0.28 \\
\hline $\begin{array}{c}\text { Body mass } \\
\text { Index }\left(\mathrm{kg} / \mathrm{m}^{2}\right)\end{array}$ & $23.6 \pm 3.9$ & $18-35$ & $26.8 \pm 3.7$ & $18-32$ & 0.09 \\
\hline
\end{tabular}

Table 3

\begin{tabular}{|c|c|c|c|c|c|c|}
\hline & \multicolumn{2}{|c|}{ Group A } & \multicolumn{2}{|c|}{ Group B } & \multirow{3}{*}{$\begin{array}{c}\mathrm{p}- \\
\text { value }\end{array}$} & \multirow{3}{*}{$\begin{array}{c}\text { Effect } \\
\text { size }\end{array}$} \\
\hline & $\begin{array}{c}\text { Baseline } \\
(\mathrm{N}=30)\end{array}$ & $\begin{array}{c}\text { Final } \\
(\mathrm{N}=27)\end{array}$ & $\begin{array}{c}\text { Baseline } \\
(\mathrm{N}=30)\end{array}$ & $\begin{array}{c}\text { Final } \\
(\mathrm{N}=28)\end{array}$ & & \\
\hline & Mean(SD) & Mean(SD) & Mean(SD) & Mean(SD) & & \\
\hline Pain $^{\mathrm{a}}$ & $8(1.5)$ & $5.0(2.5)$ & $8(1.5)$ & $3.0(2.0)$ & 0.02 & 0.81 \\
\hline FIQ & $7(2)$ & $11(3)$ & $7(2)$ & $14(2)$ & 0.01 & 0.73 \\
\hline
\end{tabular}

Table 4

\begin{tabular}{|c|c|c|c|c|c|}
\hline \multicolumn{5}{|c|}{ Socioeconomic Status } \\
\hline & & Frequency & Percent & $\begin{array}{c}\text { Valid } \\
\text { Percent }\end{array}$ & $\begin{array}{c}\text { Cumulative } \\
\text { Percent }\end{array}$ \\
\hline Valid & Middle Class & 40 & 100.0 & 100.0 & 100.0 \\
\hline \multicolumn{2}{|c|}{ a. Patients' Group = Conventional Treatment } & \\
\hline
\end{tabular}

For conventional group all patients found belonging to middle class of socioeconomics.

Table 5

\begin{tabular}{|l|c|c|c|c|c|}
\hline \multicolumn{5}{|c|}{ Gender } \\
\hline \multirow{2}{*}{} & & Frequency & Percent & $\begin{array}{c}\text { Valid } \\
\text { Percent }\end{array}$ & $\begin{array}{c}\text { Cumulative } \\
\text { Percent }\end{array}$ \\
\hline \multirow{3}{*}{ Valid } & Female & 14 & 35.0 & 35.0 & 35.0 \\
\cline { 2 - 6 } & Male & 26 & 65.0 & 65.0 & 100.0 \\
\cline { 2 - 6 } & Total & 40 & 100.0 & 100.0 & \\
\hline \multicolumn{2}{|l|}{ a. Patients' Group = Conventional Treatment } \\
\hline
\end{tabular}

In conventional group, females were 14(35\%) and males were $26(65 \%)$

Table 6

\begin{tabular}{|c|c|c|c|c|c|}
\hline \multicolumn{5}{|c|}{ Knee Pain } \\
\hline & & Frequency & Percent & $\begin{array}{c}\text { Valid } \\
\text { Percent }\end{array}$ & $\begin{array}{c}\text { Cumulative } \\
\text { Percent }\end{array}$ \\
\hline \multirow{3}{*}{ Valid } & Unilateral & 29 & 72.5 & 72.5 & 72.5 \\
\cline { 2 - 6 } & Bilateral & 11 & 27.5 & 27.5 & 100.0 \\
\cline { 2 - 6 } & Total & 40 & 100.0 & 100.0 & \\
\hline \multicolumn{2}{|c|}{ a. Patients' Group = Conventional Treatment } \\
\hline
\end{tabular}

In conventional group, 29(72.5\%) were experiencing unilateral pain, while in $11(27.5 \%)$ experiencing bilateral knee pain.

Table 7

\begin{tabular}{|c|c|c|c|c|c|}
\hline \multicolumn{5}{|c|}{ Functional Index; Walking as Far as a Mile ${ }^{\mathrm{a}}$} \\
\hline & & Frequency & Percent & $\begin{array}{c}\text { Valid } \\
\text { Percent }\end{array}$ & $\begin{array}{c}\text { Cumulative } \\
\text { Percent }\end{array}$ \\
\hline Valid & No Problem & 40 & 100.0 & 100.0 & 100.0 \\
\hline \multicolumn{2}{|c|}{ a. Patients' Group = Conventional Treatment } & \\
\hline
\end{tabular}

Conventional group all patients found with no problem while walking up to one mile

Table 8

\begin{tabular}{|c|c|c|c|c|c|}
\hline \multicolumn{6}{|c|}{ Functional Index; Climbing Up 2 Flights of Stairs (16 steps) ${ }^{\mathrm{a}}$} \\
\hline & & Frequency & Percent & \begin{tabular}{|c|} 
Valid \\
Percent
\end{tabular} & $\begin{array}{c}\text { Cumulative } \\
\text { Percent }\end{array}$ \\
\hline \multirow[t]{3}{*}{ Valid } & $\begin{array}{l}\text { Can Do with } \\
\text { Problem }\end{array}$ & 1 & 2.5 & 2.5 & 2.5 \\
\hline & No Problem & 39 & 97.5 & 97.5 & 100.0 \\
\hline & Total & 40 & 100.0 & 100.0 & \\
\hline
\end{tabular}

In conventional group, only 1(2.5\%) found doing with problem while climbing up to two flights of stairs while rest of patients found with no problem. 


\section{International Journal of Science and Research (IJSR) \\ ISSN (Online): 2319-7064 \\ Index Copernicus Value (2013): 6.14 | Impact Factor (2015): 6.391}

Table 9

\begin{tabular}{|c|c|c|c|c|c|}
\hline \multicolumn{7}{|c|}{ Functional Index; Squatting $^{\text {a }}$} \\
\hline \multirow{3}{*}{ Valid } & Frequency & Percent & $\begin{array}{c}\text { Valid } \\
\text { Percent }\end{array}$ & $\begin{array}{c}\text { Cumulative } \\
\text { Percent }\end{array}$ \\
\cline { 2 - 6 } & Problem & 3 & 7.5 & 7.5 & 7.5 \\
\cline { 2 - 6 } & No Problem & 37 & 92.5 & 92.5 & 100.0 \\
\cline { 2 - 6 } & \multicolumn{7}{|c|}{ Total } & 40 & 100.0 & 100.0 & \\
\hline \multicolumn{7}{|c|}{ a. Patients' Group = Conventional Treatment } & & \\
\hline
\end{tabular}

In conventional group, 3(7.5\%) found doing squat with problem while rest of 37(92.5\%) found without problem.

Table 10

\begin{tabular}{|c|c|c|c|c|c|}
\hline \multicolumn{7}{|c|}{ Functional Index; Kneeling $^{\text {a }}$} \\
\hline & & Frequency & Percent & $\begin{array}{c}\text { Valid } \\
\text { Percent }\end{array}$ & $\begin{array}{c}\text { Cumulative } \\
\text { Percent }\end{array}$ \\
\hline \multirow{3}{*}{ Valid } & $\begin{array}{c}\text { Can Do with } \\
\text { Problem }\end{array}$ & 10 & 25.0 & 25.0 & 25.0 \\
\cline { 2 - 6 } & No Problem & 30 & 75.0 & 75.0 & 100.0 \\
\cline { 2 - 6 } & \multicolumn{7}{|c|}{ Total } & 40 & 100.0 & 100.0 & \\
\hline
\end{tabular}

In conventional group, 10(25.0) patients had problem in kneeling, while 30(75\%) had no problem

Table 11

\begin{tabular}{|c|c|c|c|c|c|}
\hline \multicolumn{6}{|c|}{$\begin{array}{l}\text { Functional Index; Sitting for Prolonged Periods with } \\
\text { Your Knees Bent in One Position }\end{array}$} \\
\hline & & Frequency & Percent & $\begin{array}{c}\text { Valid } \\
\text { Percent }\end{array}$ & $\begin{array}{l}\text { Cumulative } \\
\text { Percent }\end{array}$ \\
\hline \multirow[t]{3}{*}{ Valid } & $\begin{array}{l}\text { Can Do with } \\
\text { Problem }\end{array}$ & 19 & 47.5 & 47.5 & 47.5 \\
\hline & No Problem & 21 & 52.5 & 52.5 & 100.0 \\
\hline & Total & 40 & 100.0 & 100.0 & \\
\hline \multicolumn{4}{|c|}{ a. Patients' Group = Conventional Treatmen } & & \\
\hline
\end{tabular}

In conventional group, sitting for prolonged periods with knees bent in one position, 19(47.5\%) found problematic while 21(52.5\%) found without problem.

Table 12

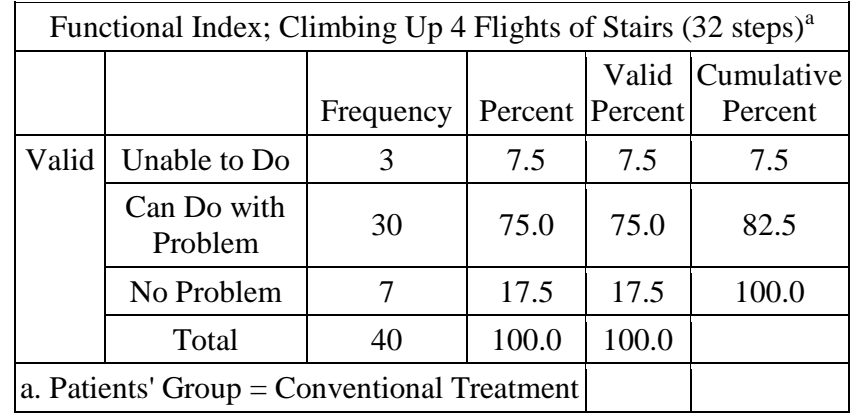

In conventional group, climbing up 4 flights of stairs, $3(7.5 \%)$ found unable to do, 30(75\%) found with problem while $7(71.5 \%)$ found without problem.
Table 13

\begin{tabular}{|c|c|c|c|c|c|}
\hline \multicolumn{6}{|c|}{$\begin{array}{l}\text { Functional Index; Running a Short Distance, Say } \\
100 \text { Meters. (About the Length of a Football Field) }\end{array}$} \\
\hline & & Frequency & Percent & $\begin{array}{c}\text { Valid } \\
\text { Percent }\end{array}$ & $\begin{array}{c}\text { Cumulative } \\
\text { Percent }\end{array}$ \\
\hline \multirow[t]{3}{*}{ Valid } & Unable to Do & 18 & 45.0 & 45.0 & 45.0 \\
\hline & $\begin{array}{l}\text { Can Do with } \\
\text { Problem }\end{array}$ & 22 & 55.0 & 55.0 & 100.0 \\
\hline & Total & 40 & 100.0 & 100.0 & \\
\hline
\end{tabular}

In conventional group, running a short distance up to 100 meters, 18(45\%) were unable to do, 22(55\%) were doing with problem.

Table 14

\begin{tabular}{|c|c|c|c|c|c|}
\hline \multicolumn{6}{|c|}{$\begin{array}{l}\text { Functional Index; Walking a Short } \\
\text { Distance (About a City Block) }\end{array}$} \\
\hline & & Frequency & Percent & \begin{tabular}{|c|} 
Valid \\
Percent \\
\end{tabular} & $\begin{array}{c}\text { Cumulative } \\
\text { Percent }\end{array}$ \\
\hline \multirow[t]{3}{*}{ Valid } & Unable to Do & 33 & 82.5 & 82.5 & 82.5 \\
\hline & $\begin{array}{l}\text { Can Do with } \\
\text { Problem }\end{array}$ & 7 & 17.5 & 17.5 & 100.0 \\
\hline & Total & 40 & 100.0 & 100.0 & \\
\hline
\end{tabular}

In conventional group, walking a short distance, say a city block, 33(82.5\%) found unable to do while 7(17.5\%) found doing it with problem.

Table 15

\begin{tabular}{|l|c|c|c|c|c|}
\hline \multicolumn{6}{|c|}{ SocioecnomicStatus $^{\mathrm{a}}$} \\
\hline & & Frequency & Percent & $\begin{array}{c}\text { Valid } \\
\text { Percent }\end{array}$ & $\begin{array}{c}\text { Cumulative } \\
\text { Percent }\end{array}$ \\
\hline \multirow{2}{*}{ Valid } & Lower Class & 1 & 2.5 & 2.5 & 2.5 \\
\cline { 2 - 6 } & Middle Class & 39 & 97.5 & 97.5 & 100.0 \\
\cline { 2 - 6 } & \multicolumn{7}{|c|}{ Total } & 40 & 100.0 & 100.0 & \\
\hline \multicolumn{2}{|c|}{ a. Patients' Group = Pain Release Phenomenon } \\
\hline
\end{tabular}

For pain release phenomenon group, 1(2.5\%) patients were from lower class of socioeconomics while 39(97.5\%) belonging to middle class of socioeconomics.

Table 16

\begin{tabular}{|c|c|c|c|c|c|}
\hline \multicolumn{7}{|c|}{} & Frequency & Percent & $\begin{array}{c}\text { Valid } \\
\text { Percent }\end{array}$ & $\begin{array}{c}\text { Cumulative } \\
\text { Percent }\end{array}$ \\
\hline \multirow{3}{*}{ Valid } & Female & 16 & 40.0 & 40.0 & 40.0 \\
\cline { 2 - 6 } & Male & 24 & 60.0 & 60.0 & 100.0 \\
\cline { 2 - 6 } & Total & 40 & 100.0 & 100.0 & \\
\hline
\end{tabular}

In pain release phenomenon group, females were $16(40 \%)$ and males were $24(60 \%)$ 


\section{International Journal of Science and Research (IJSR) \\ ISSN (Online): 2319-7064}

Index Copernicus Value (2013): 6.14 | Impact Factor (2015): 6.391

Table 17

\begin{tabular}{|l|l|l|l|l|l|}
\hline \multicolumn{5}{|l|}{ Knee Pain ${ }^{\mathrm{a}}$} \\
\hline & & Frequency & Percent & $\begin{array}{l}\text { Valid } \\
\text { Percent }\end{array}$ & $\begin{array}{l}\text { Cumulative } \\
\text { Percent }\end{array}$ \\
\hline \multirow{3}{*}{ Valid } & Unilateral & 30 & 75.0 & 75.0 & 75.0 \\
\cline { 2 - 6 } & Bilateral & 9 & 22.5 & 22.5 & 97.5 \\
\cline { 2 - 6 } & Total & 40 & 100.0 & 100.0 & \\
\hline \multicolumn{6}{|l|}{ a. Patients' Group = Pain Release Phenomenon } \\
\hline
\end{tabular}

In pain release phenomenon group, 30(75\%) were experiencing unilateral pain, while in 9(22.5\%) experiencing bilateral knee pain.

Table 18

\begin{tabular}{|l|l|l|l|l|l|}
\hline \multicolumn{5}{|c|}{ Functional Index; Walking as Far as a Mile } \\
\hline & & Frequency & Percent & $\begin{array}{l}\text { Valid } \\
\text { Percent }\end{array}$ & $\begin{array}{l}\text { Cumulative } \\
\text { Percent }\end{array}$ \\
\hline \multirow{3}{*}{ Valid } & $\begin{array}{l}\text { Can Do with } \\
\text { Problem }\end{array}$ & 2 & 5.0 & 5.0 & 5.0 \\
\cline { 2 - 6 } & No Problem & 38 & 95.0 & 95.0 & 100.0 \\
\cline { 2 - 6 } & \multicolumn{7}{|l|}{ Total } & 40 & 100.0 & 100.0 & \\
\hline $\begin{array}{l}\text { a. Patients' Group = Pain Release } \\
\text { Phenomenon }\end{array}$ & & \\
\hline
\end{tabular}

Pain release phenomenon group, 2(5\%) patients found walking a mile with problem while $38(95 \%)$ found without problem.

Table 19

Functional Index; Climbing Up 2 Flights of Stairs (16 steps) ${ }^{\mathrm{a}}$

\begin{tabular}{|l|l|l|l|l|l|}
\hline & & Frequency & Percent & $\begin{array}{l}\text { Valid } \\
\text { Percent }\end{array}$ & $\begin{array}{l}\text { Cumulative } \\
\text { Percent }\end{array}$ \\
\hline \multirow{7}{*}{ Valid } & $\begin{array}{l}\text { Can Do with } \\
\text { Problem }\end{array}$ & 2 & 5.0 & 5.0 & 5.0 \\
\cline { 2 - 6 } & No Problem & 38 & 95.0 & 95.0 & 100.0 \\
\cline { 2 - 6 } & Total & 40 & 100.0 & 100.0 & \\
\hline $\begin{array}{l}\text { a.Patients' Group } \\
\text { Phenomenon }\end{array}$ & Pain Release & & \\
\hline
\end{tabular}

In pain release phenomenon group, only 2(5\%) found doing with problem while climbing up to two flights of stairs while rest of patients found with no problem.

Table 20

\begin{tabular}{|l|l|l|l|l|l|}
\hline \multicolumn{5}{|l|}{ Functional Index; Squatting } \\
\hline
\end{tabular}

In pain release phenomenon group, $13(32.5 \%)$ found doing squat with problem while rest of $27(67.5 \%)$ found without problem.
Table 21

\begin{tabular}{|c|c|c|c|c|c|}
\hline \multicolumn{6}{|c|}{ Functional Index; Kneeling } \\
\hline & Frequency & Percent & $\begin{array}{c}\text { Valid } \\
\text { Percent }\end{array}$ & $\begin{array}{c}\text { Cumulative } \\
\text { Percent }\end{array}$ \\
\hline Valid & $\begin{array}{c}\text { Can Do with } \\
\text { Problem }\end{array}$ & 22 & 55.0 & 55.0 & 55.0 \\
\cline { 2 - 6 } & No Problem & 18 & 45.0 & 45.0 & 100.0 \\
\cline { 2 - 6 } & Total & 40 & 100.0 & 100.0 & \\
\hline \multicolumn{7}{|c|}{$\begin{array}{c}\text { a. Patients' Group = Pain Release } \\
\text { Phenomenon }\end{array}$} & & \\
\hline
\end{tabular}

In pain release phenomenon group, 22(55.0) patients had problem in kneeling, while $18(45 \%)$ had no problem.

Table 22

Functional Index; Sitting for Prolonged Periods with Your Knees Bent in One Position ${ }^{\mathrm{a}}$

\begin{tabular}{|c|c|c|c|c|c|}
\hline & Frequency & Percent & $\begin{array}{c}\text { Valid } \\
\text { Percent }\end{array}$ & $\begin{array}{c}\text { Cumulative } \\
\text { Percent }\end{array}$ \\
\hline Valid & $\begin{array}{c}\text { Can Do with } \\
\text { Problem }\end{array}$ & 28 & 70.0 & 70.0 & 70.0 \\
\cline { 2 - 6 } & No Problem & 12 & 30.0 & 30.0 & 100.0 \\
\cline { 2 - 6 } & Total & 40 & 100.0 & 100.0 & \\
\hline \multicolumn{3}{|c|}{$\begin{array}{c}\text { a. Patients' Group = Pain Release } \\
\text { Phenomenon }\end{array}$} & & \\
\hline
\end{tabular}

In pain release phenomenon group, sitting for prolonged periods with knees bent in one position, 28(70\%) found problematic while $12(30 \%)$ found without problem.

Table 23

\begin{tabular}{|c|c|c|c|c|c|}
\hline \multicolumn{6}{|c|}{ Functional Index; Climbing Up 4 Flights of Stairs (32 steps) } \\
\hline & & Frequency & Percent & \begin{tabular}{|c|} 
Valid \\
Percent
\end{tabular} & $\begin{array}{c}\text { Cumulative } \\
\text { Percent }\end{array}$ \\
\hline \multirow[t]{4}{*}{ Valid } & Unable to Do & 9 & 22.5 & 22.5 & 22.5 \\
\hline & $\begin{array}{l}\text { Can Do with } \\
\text { Problem }\end{array}$ & 28 & 70.0 & 70.0 & 92.5 \\
\hline & No Problem & 3 & 7.5 & 7.5 & 100.0 \\
\hline & Total & 40 & 100.0 & 100.0 & \\
\hline \multicolumn{4}{|c|}{$\begin{array}{c}\text { a. Patients' Group = Pain Release } \\
\text { Phenomenon }\end{array}$} & & \\
\hline
\end{tabular}

In pain release phenomenon group, climbing up 4 flights of stairs, 4(22.5\%) found unable to do, 28(70\%) found with problem while $3(7.5 \%)$ found without problem.

Table 24

Functional Index; Running a Short Distance, Say 100 Meters. (About the Length of a Football Field) ${ }^{\mathrm{a}}$

\begin{tabular}{|c|c|c|c|c|c|}
\hline & & Frequency & Percent & $\begin{array}{c}\text { Valid } \\
\text { Percent }\end{array}$ & $\begin{array}{c}\text { Cumulative } \\
\text { Percent }\end{array}$ \\
\hline \multirow[t]{4}{*}{ Valid } & Unable to Do & 27 & 67.5 & 67.5 & 67.5 \\
\hline & $\begin{array}{l}\text { Can Do with } \\
\text { Problem }\end{array}$ & 12 & 30.0 & 30.0 & 97.5 \\
\hline & No Problem & 1 & 2.5 & 2.5 & 100.0 \\
\hline & Total & 40 & 100.0 & 100.0 & \\
\hline \multicolumn{4}{|c|}{$\begin{array}{c}\text { a. Patients' Group = Pain Release } \\
\text { Phenomenon }\end{array}$} & & \\
\hline
\end{tabular}

In pain release phenomenon group, running a short distance up to 100 meters, $27(67.5 \%)$ were unable to do, $12(30 \%)$

\section{Volume 5 Issue 7, July 2016 www.ijsr.net}




\section{International Journal of Science and Research (IJSR) \\ ISSN (Online): 2319-7064}

Index Copernicus Value (2013): 6.14 | Impact Factor (2015): 6.391

were doing with problem, rest of $1(205 \%)$ found without problem.

\section{Table 25}

\begin{tabular}{|c|c|c|c|c|c|}
\hline \multicolumn{6}{|c|}{$\begin{array}{l}\text { Functional Index; Walking a Short } \\
\text { Distance (About a City Block) }\end{array}$} \\
\hline & & Frequency & Percent & $\begin{array}{l}\text { Valid } \\
\text { Percent }\end{array}$ & $\begin{array}{c}\text { Cumulative } \\
\text { Percent }\end{array}$ \\
\hline \multirow[t]{3}{*}{ Valid } & Unable to Do & 33 & 82.5 & 82.5 & 82.5 \\
\hline & $\begin{array}{l}\text { Can Do with } \\
\text { Problem }\end{array}$ & 7 & 17.5 & 17.5 & 100.0 \\
\hline & Total & 40 & 100.0 & 100.0 & \\
\hline \multicolumn{4}{|c|}{$\begin{array}{c}\text { a. } \text { Patients' Group = Pain Release } \\
\text { Phenomenon }\end{array}$} & & \\
\hline
\end{tabular}

In pain release phenomenon group, walking a short distance, say a city block, 33(82.5\%) found unable to do while $7(17.5 \%)$ found doing it with problem.

\section{List of Figure}

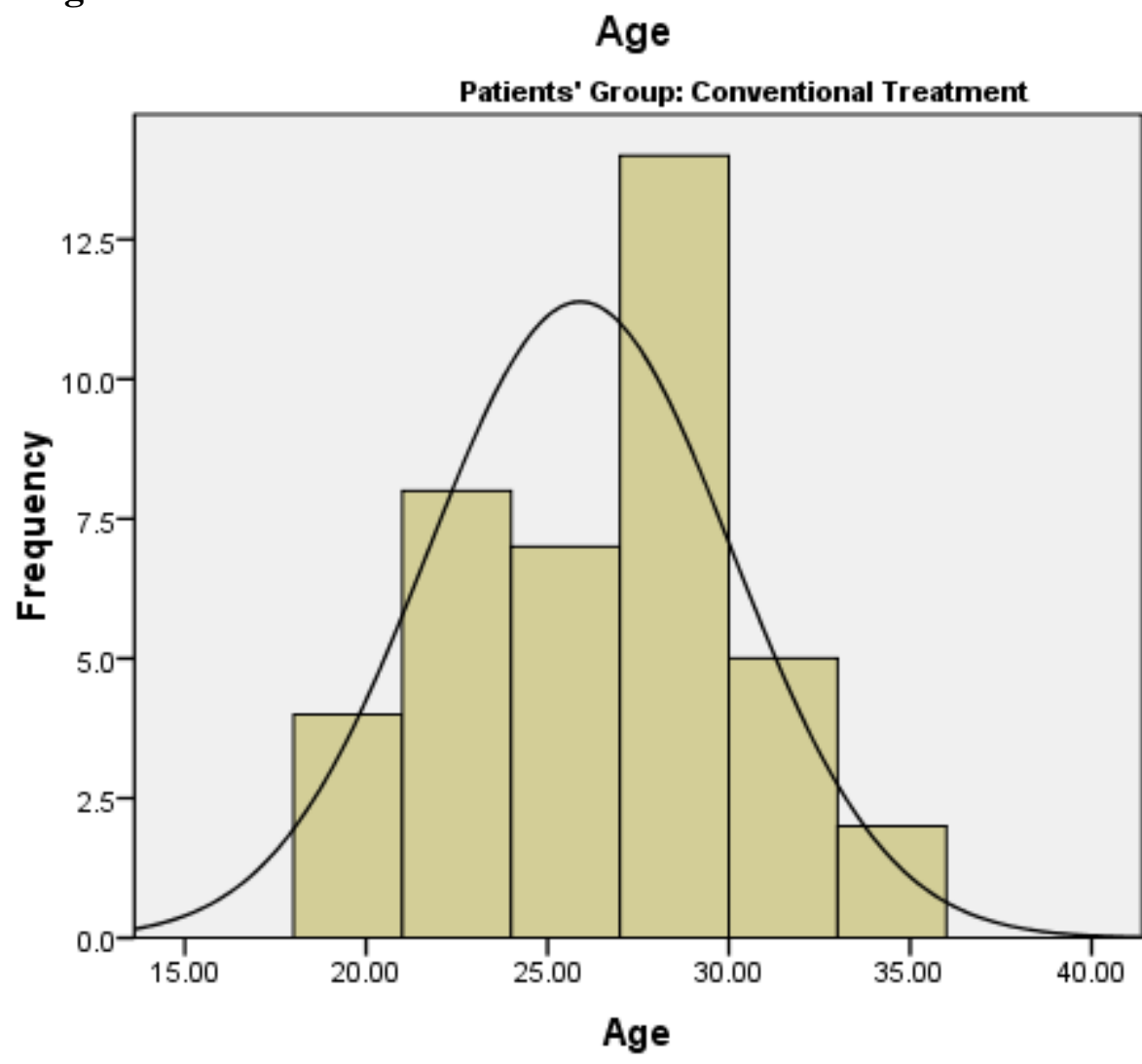

Mean $=25.90$ Std. Dev. $=4.205$

$\mathrm{N}=40$

Figure 1

The mean age of patients in conventional group found to be 25.8(SD+4.205) on histogram with normal curve, while the curve negatively skewed towards lesser values. 


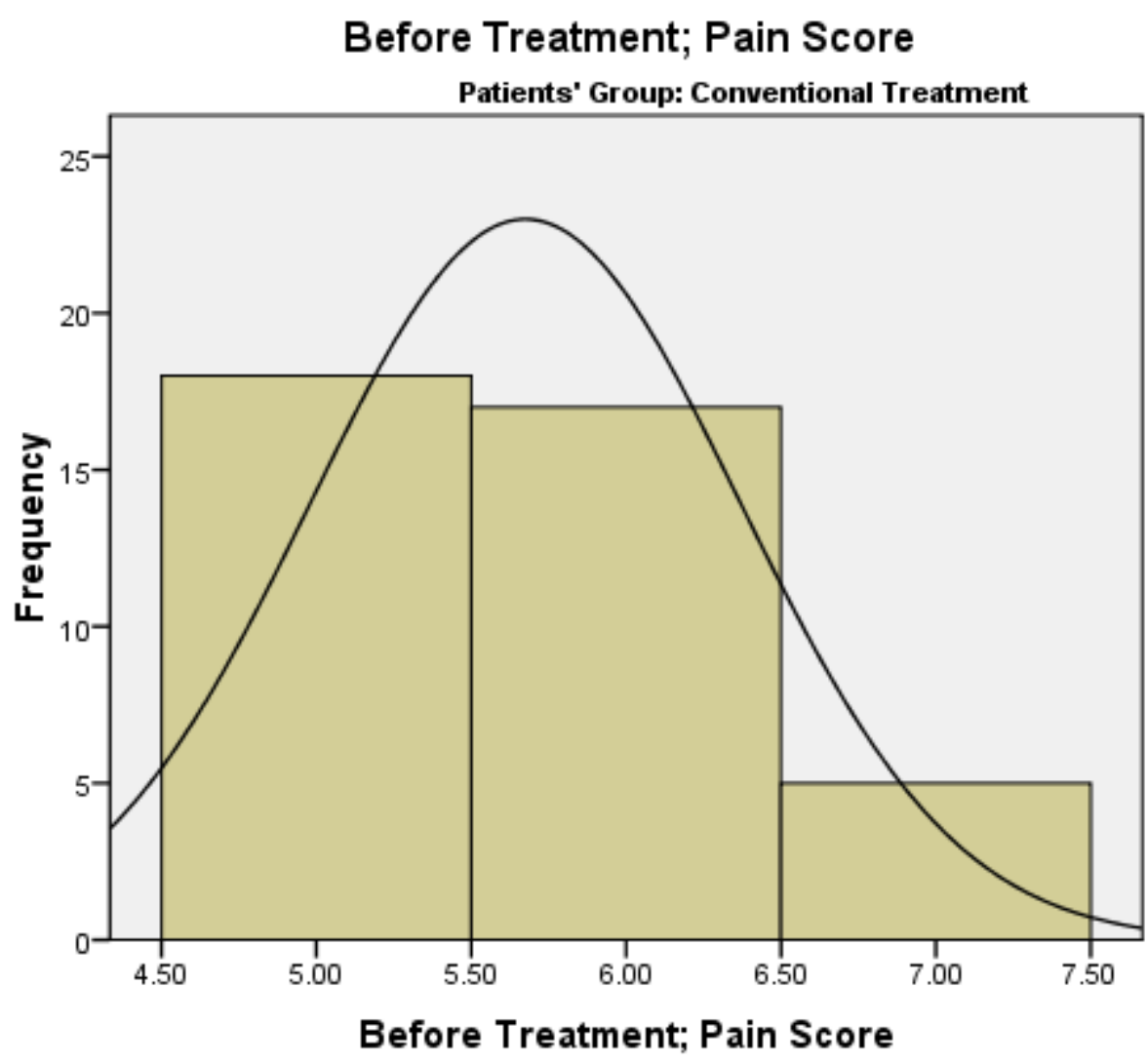

Mean $=5.68$

Figure 2

For conventional group, before treatment pain score histogram with normal curve showed a mean of 5.68(SD \pm 0.694$)$, while curve skewed negatively towards lesser values.

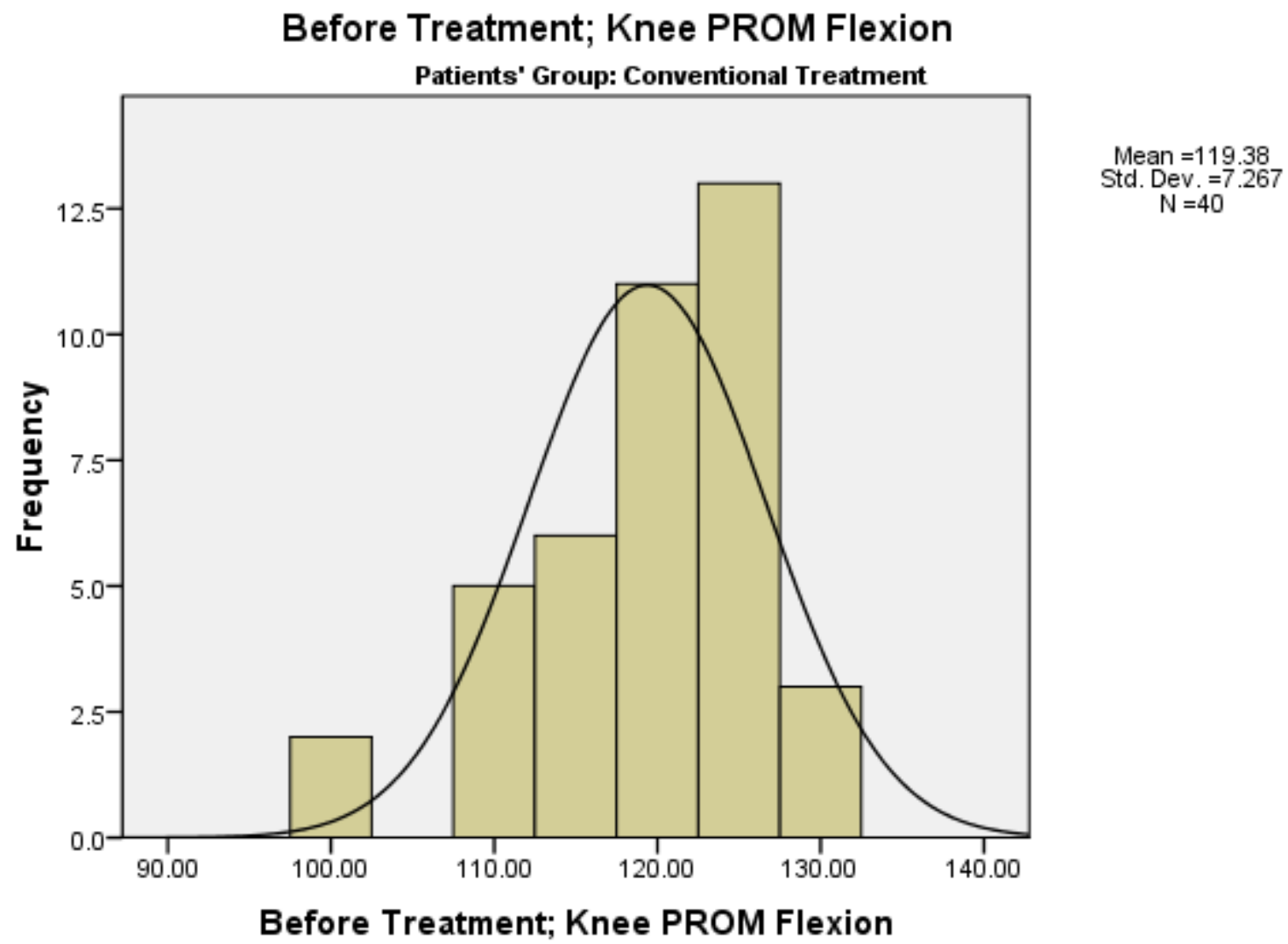

Figure 3

For conventional group, knee PROM flexion range before treatment found to be 119.38 on average with standard deviation 7.267, while curve normal distributed.

Volume 5 Issue 7, July 2016 www.ijsr.net 


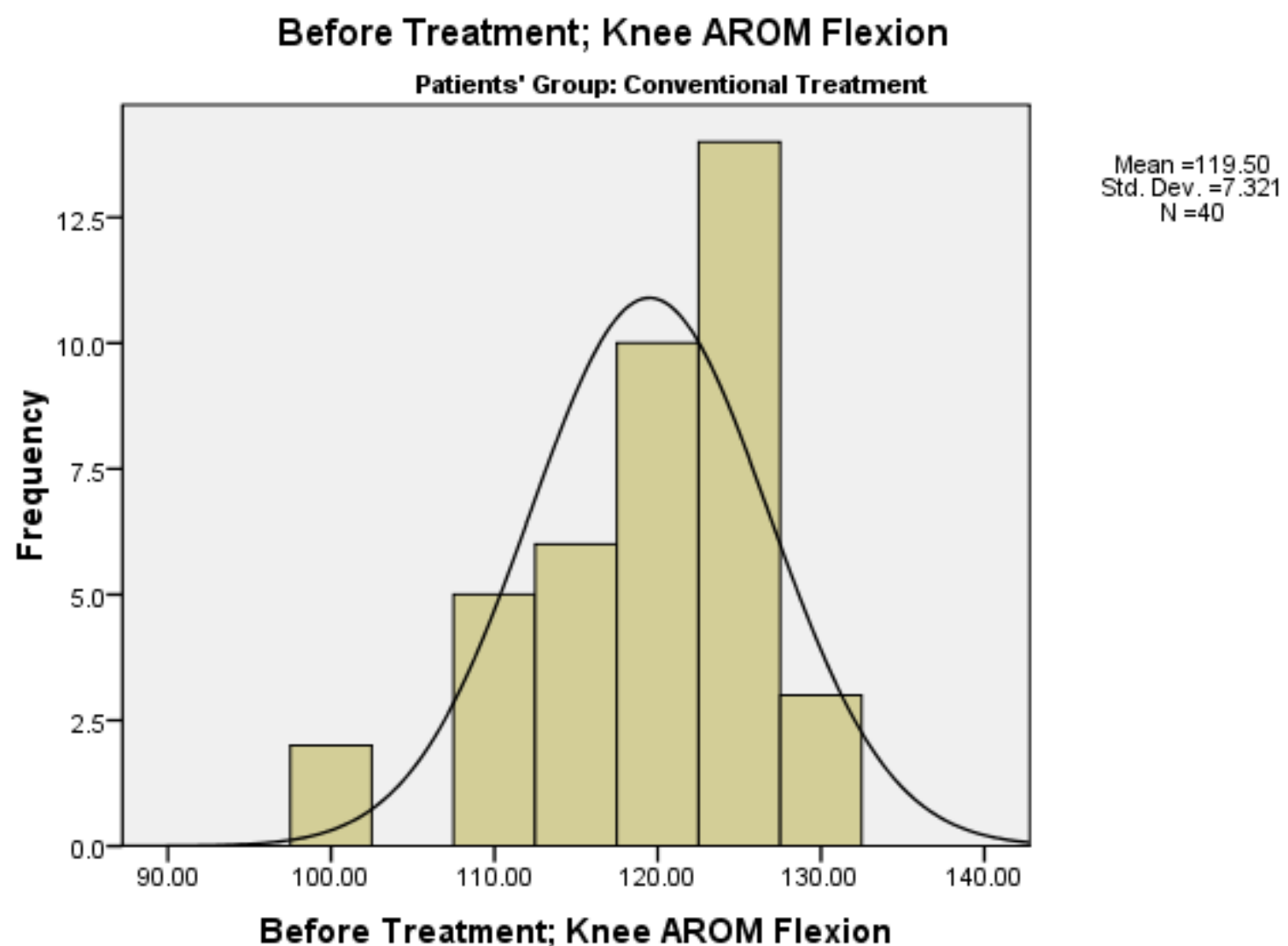

Figure 4

For conventional group, knee AROM flexion range before treatment found to be 119.38 on average with standard deviation 7.267, while curve normal distributed.

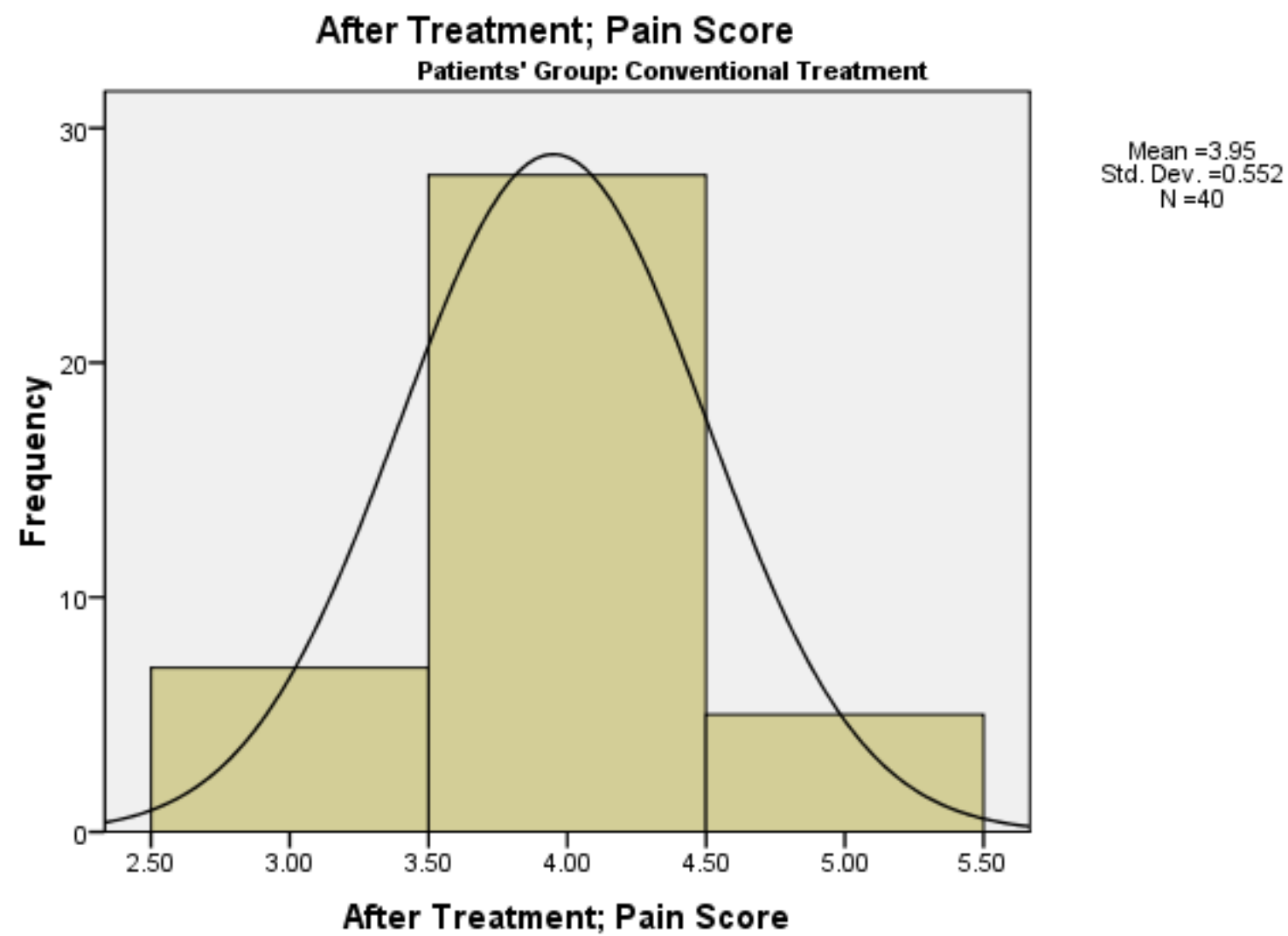

Figure 5

For conventional group, after treatment pain score histogram with normal curve showed a mean of 3.95(SD+0.562), while curve normally distributed.

Volume 5 Issue 7, July 2016 www.ijsr.net 


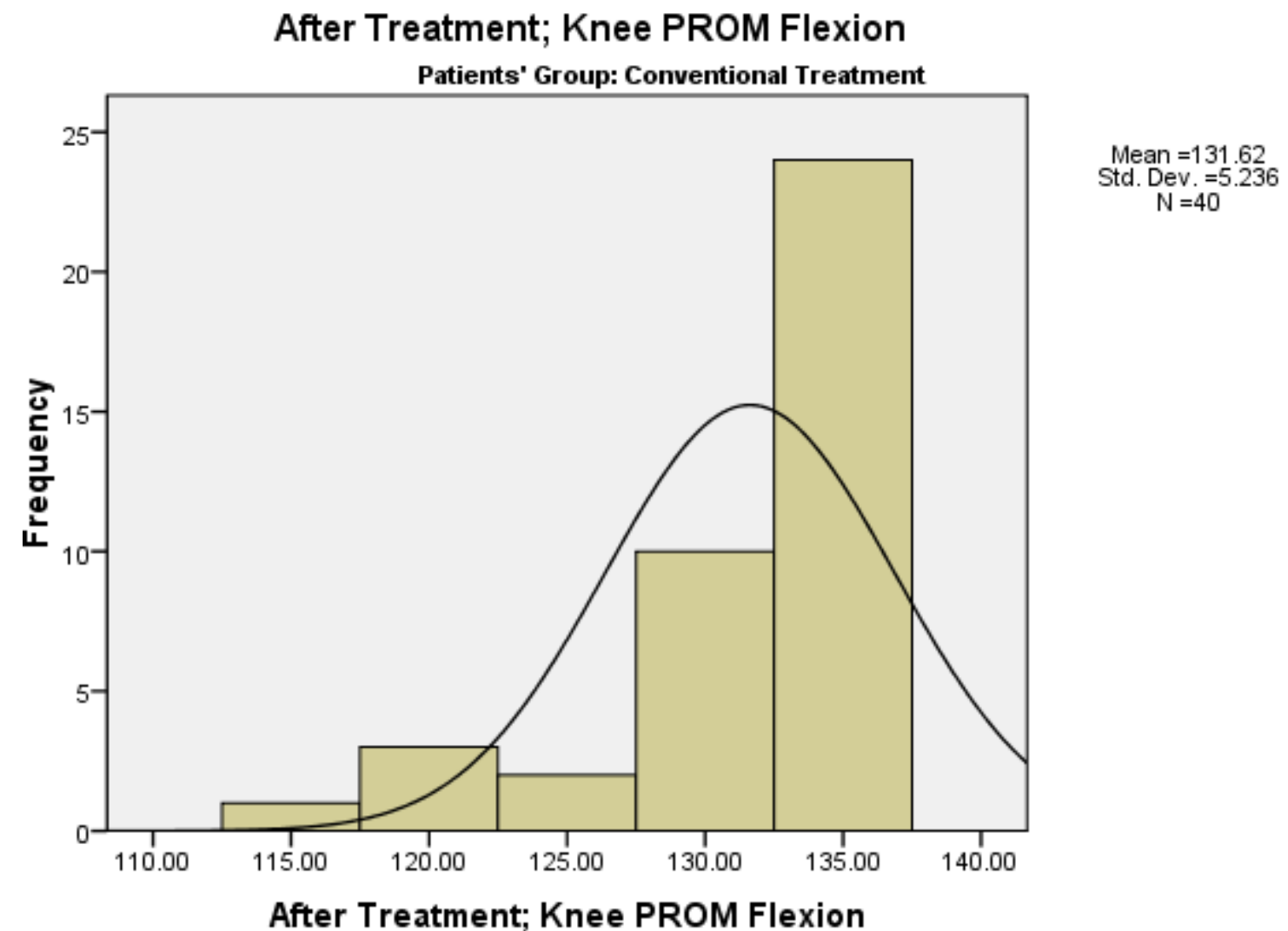

Figure 6

For conventional group, knee PROM flexion range after treatment found to be 131.62 on average with standard deviation 5.236, while curve normal distributed.

After Treatment; Knee AROM Flexion

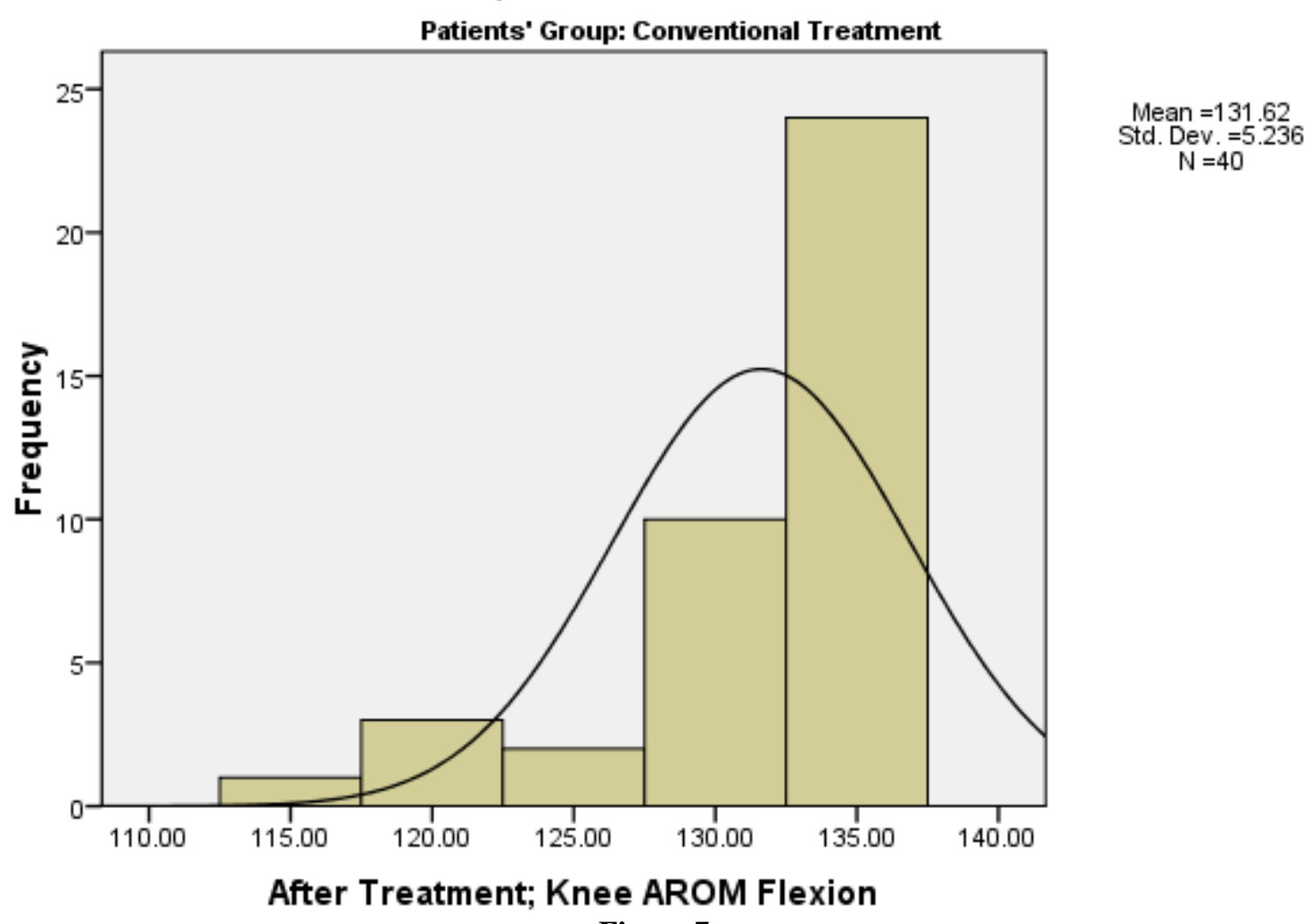

Figure 7

For conventional group, knee AROM flexion range before treatment found to be 131.62 on average with standard deviation 5.236, while curve normal distributed.

Volume 5 Issue 7, July 2016 www.ijsr.net 


\section{International Journal of Science and Research (IJSR) \\ ISSN (Online): 2319-7064}

Index Copernicus Value (2013): 6.14 | Impact Factor (2015): 6.391

\section{Total Score: Functional Index Questionnaire}

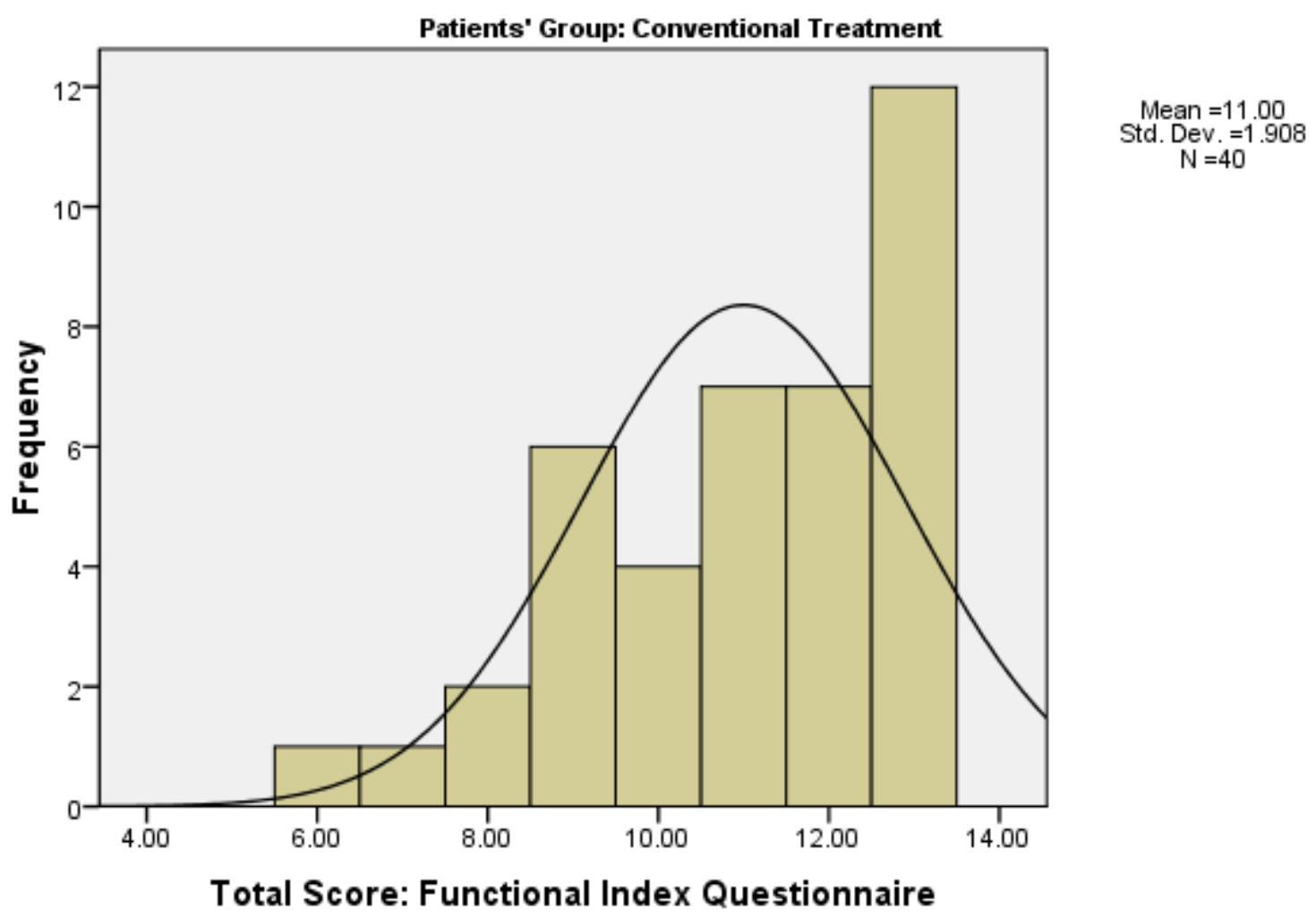

Figure 8

For conventional group, total score of Functional Index Questionnaire found to be 11.0 on average with standard deviation 1.908, while curve skewed positively towards higher values.

\section{Performance Level: Functional Index Questionnaire}

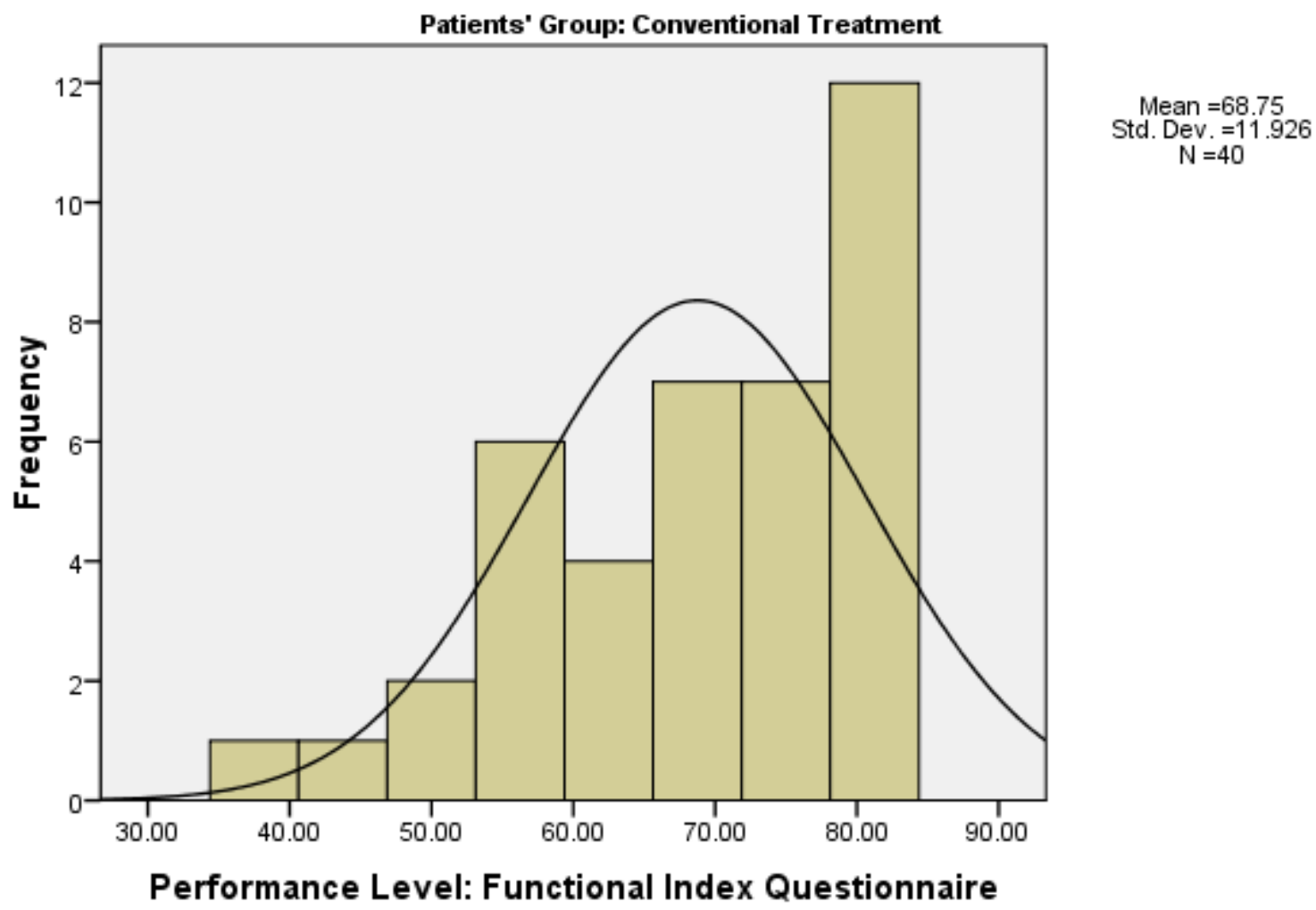

Figure 9

For conventional group, performance level of Functional Index Questionnaire found to be 68.75 on average with standard deviation 11.926, while curve skewed positively towards higher values.

Volume 5 Issue 7, July 2016 www.ijsr.net 


\section{International Journal of Science and Research (IJSR) \\ ISSN (Online): 2319-7064}

Index Copernicus Value (2013): 6.14 | Impact Factor (2015): 6.391

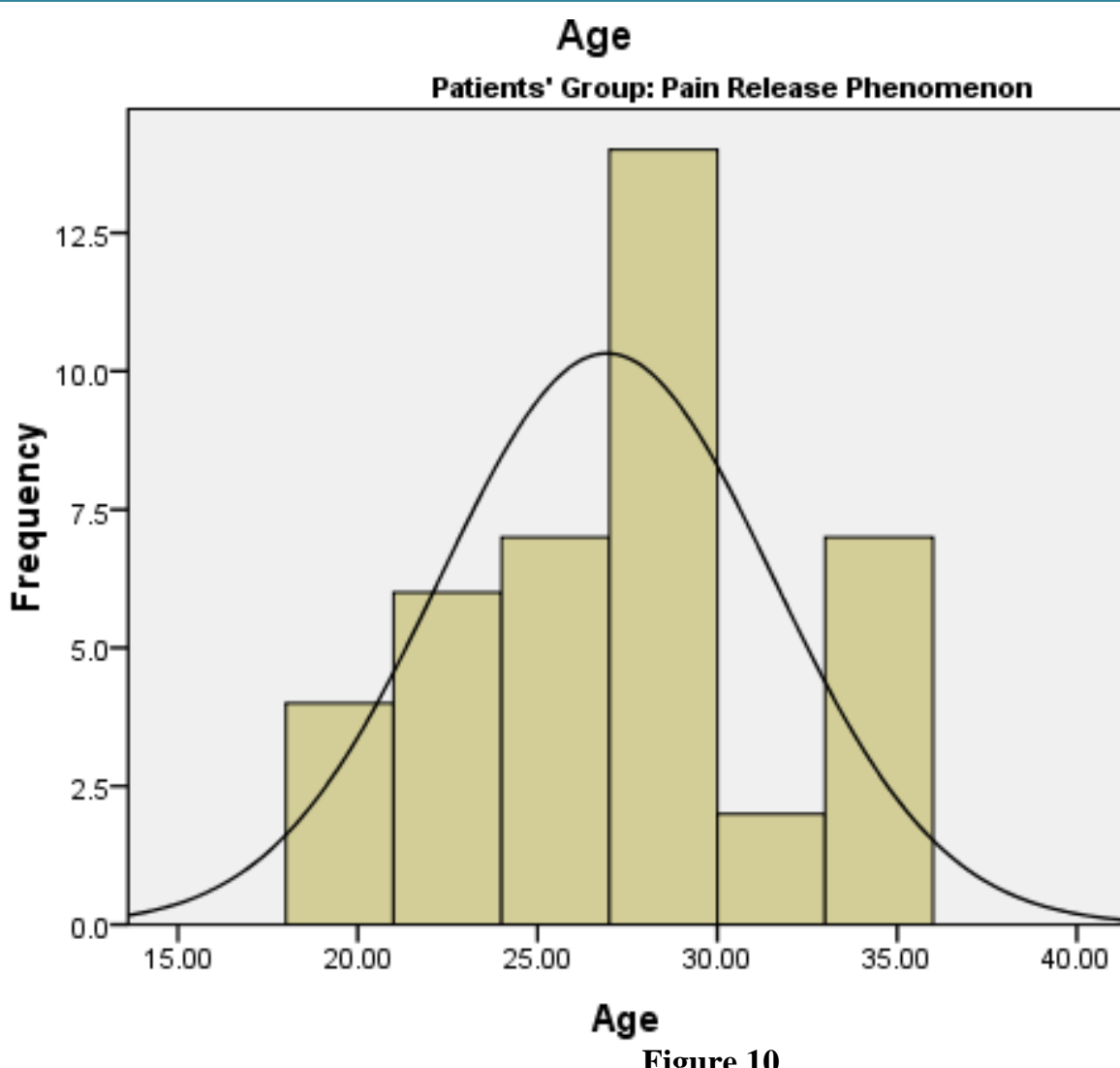

Mean $=26.92$

Std. Dev. $=4.638$

$\mathrm{N}=40$

Figure 10

The mean age of patients in pain release phenomenon group found to be 26.92(SD+4.638) on histogram with normal curve, while the curve negatively skewed towards lesser values.

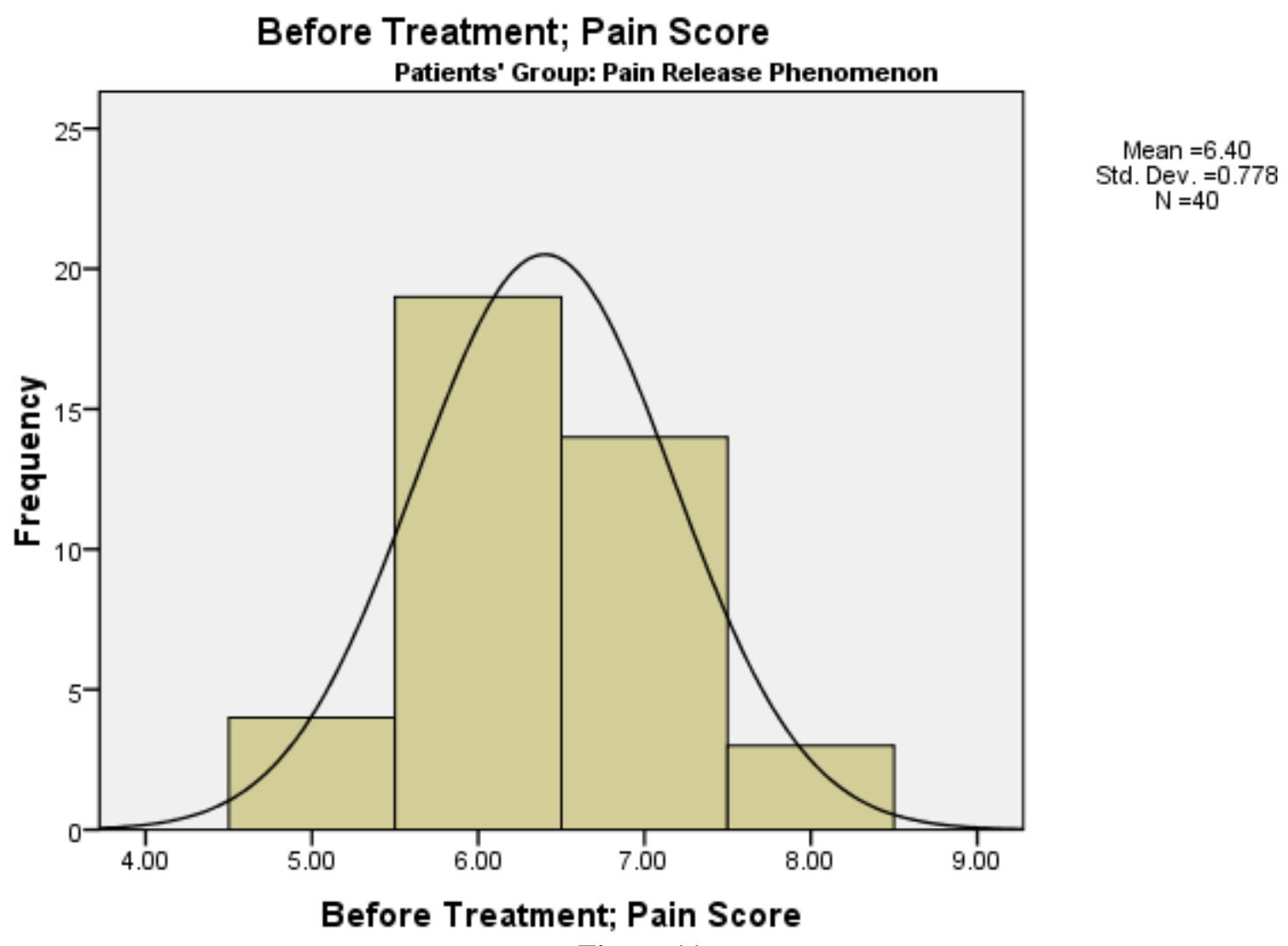

Figure 11

For pain release phenomenon group, before treatment pain score histogram with normal curve showed a mean of 5.68(SD+0.694), while curve skewed negatively towards lesser values.

Volume 5 Issue 7, July 2016 www.ijsr.net

Licensed Under Creative Commons Attribution CC BY 
International Journal of Science and Research (IJSR)

ISSN (Online): 2319-7064

Index Copernicus Value (2013): 6.14 | Impact Factor (2015): 6.391

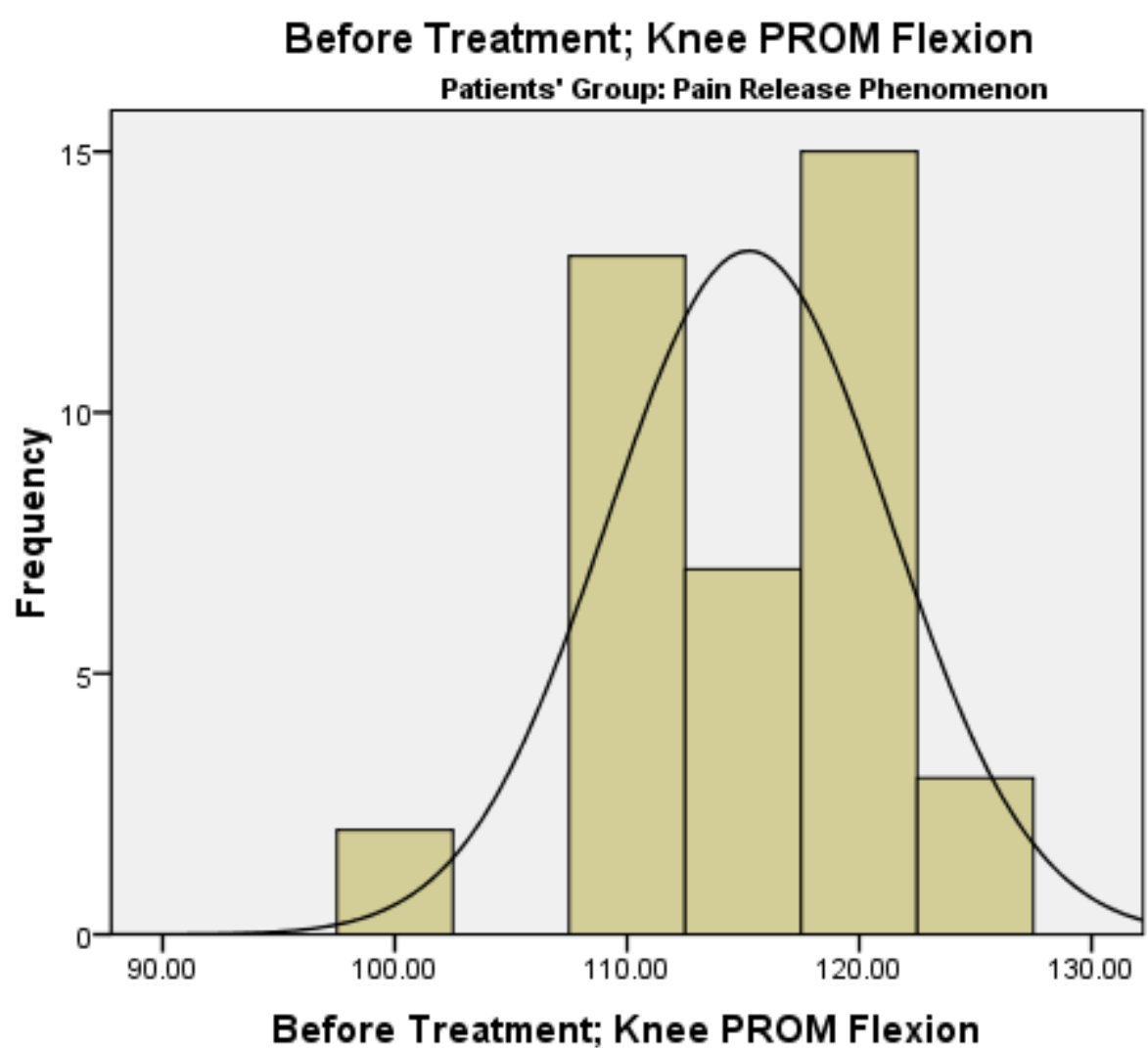

Mean $=115.25$

Std. Dev. $=6.092$

$\mathrm{N}=40$

Figure 12

For pain release phenomenon group, knee PROM flexion range before treatment found to be 119.38 on average with standard deviation 7.267, while curve normal distributed.

\section{Before Treatment; Knee AROM Flexion}

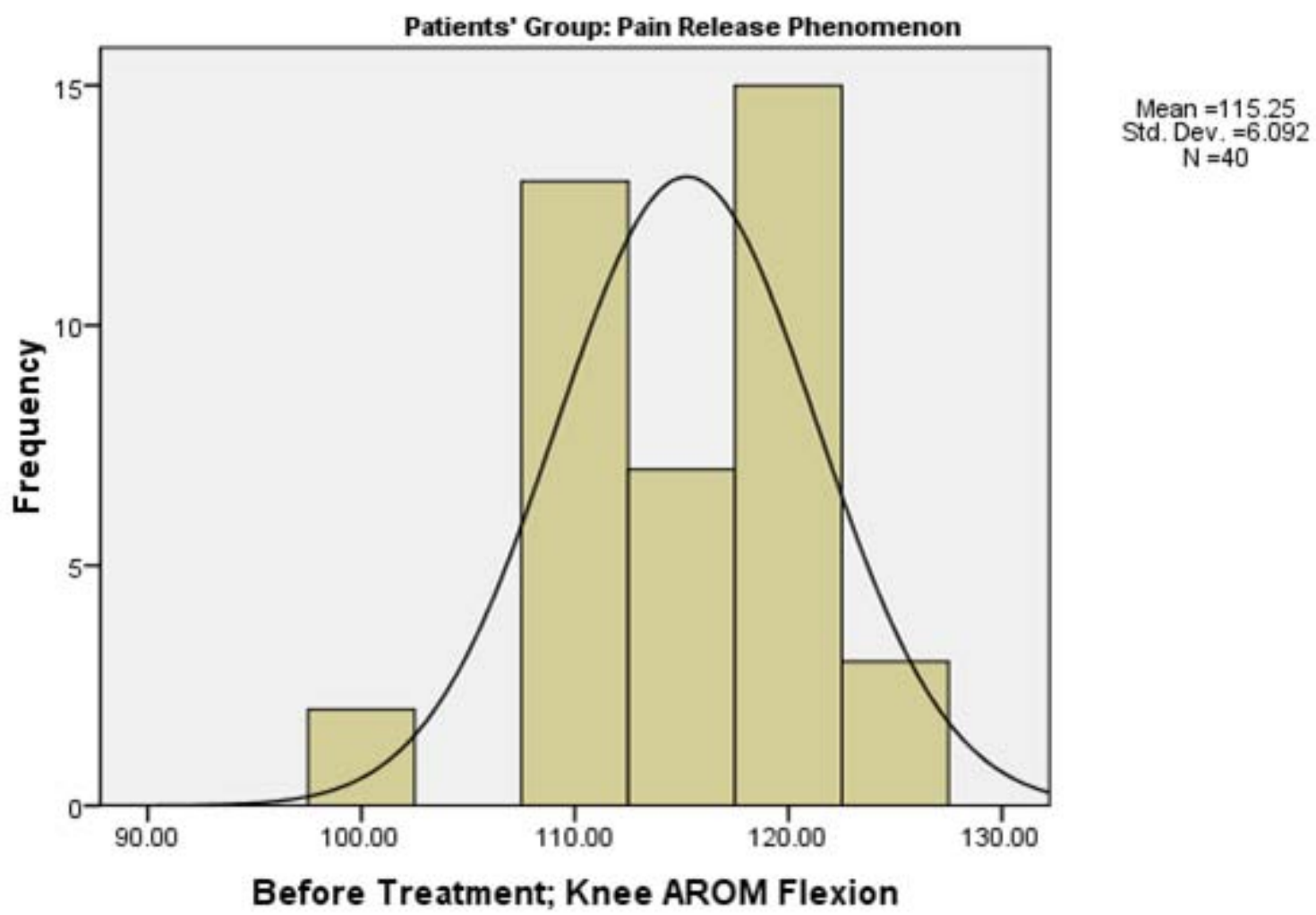

Figure 13

For pain release phenomenon group, knee AROM flexion range before treatment found to be 119.38 on average with standard deviation 7.267, while curve normal distributed.

Volume 5 Issue 7, July 2016 www.ijsr.net 


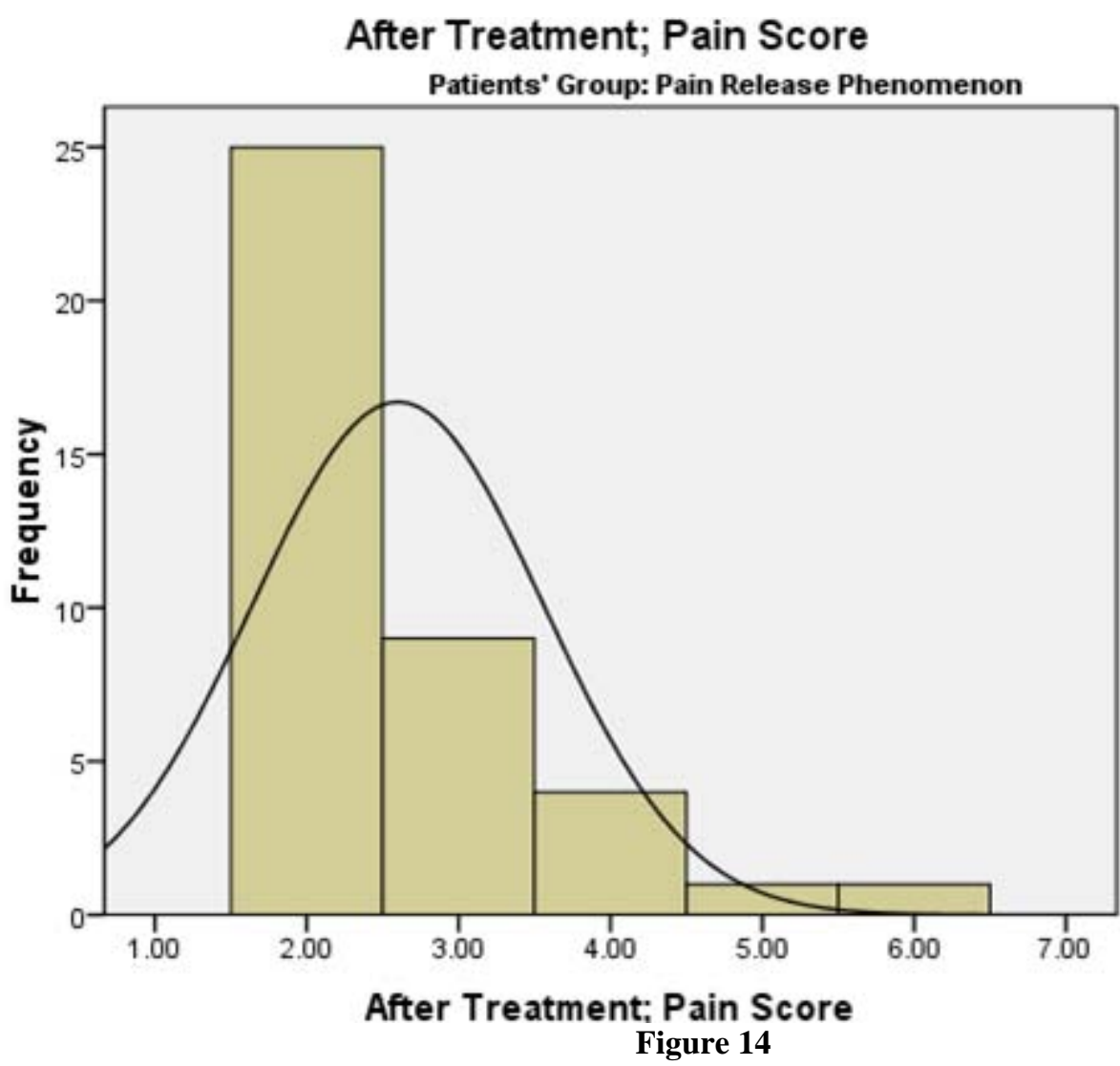

Mean $=2.60$

Std. Dev. $=0.955$

$\mathrm{N}=40$

For pain release phenomenon group, after treatment pain score histogram with normal curve showed a mean of 2.60(SD+0.955), while curve normally distributed.

After Treatment; Knee PROM Flexion

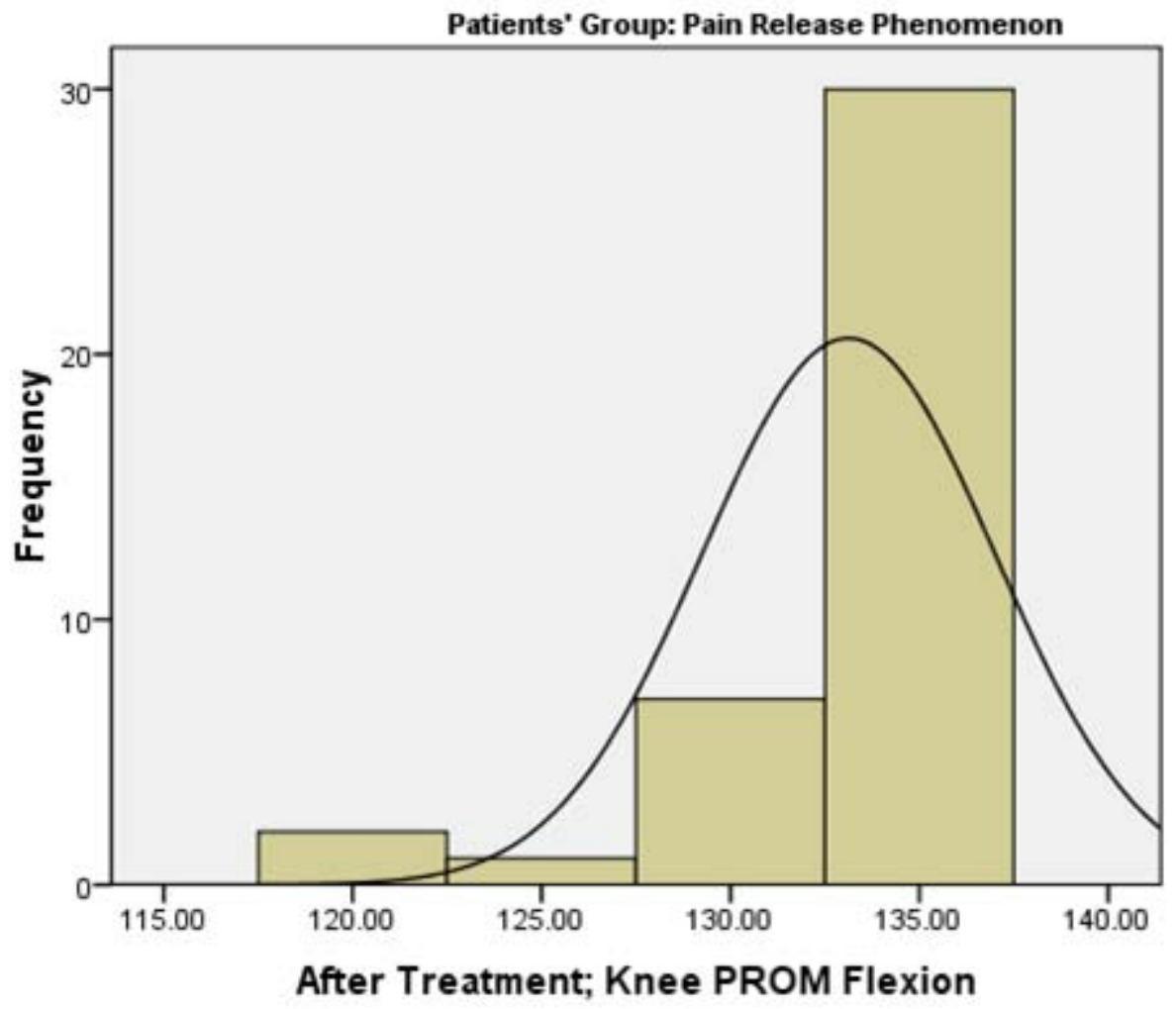

Mean $=133.12$

Std. Dev, $=3.871$ $\mathrm{N}=40$

Figure 15

For pain release phenomenon group, knee PROM flexion range after treatment found to be 133.12 on average with standard deviation 3.871, while curve skewed positively.

Volume 5 Issue 7, July 2016 www.ijsr.net

Licensed Under Creative Commons Attribution CC BY 


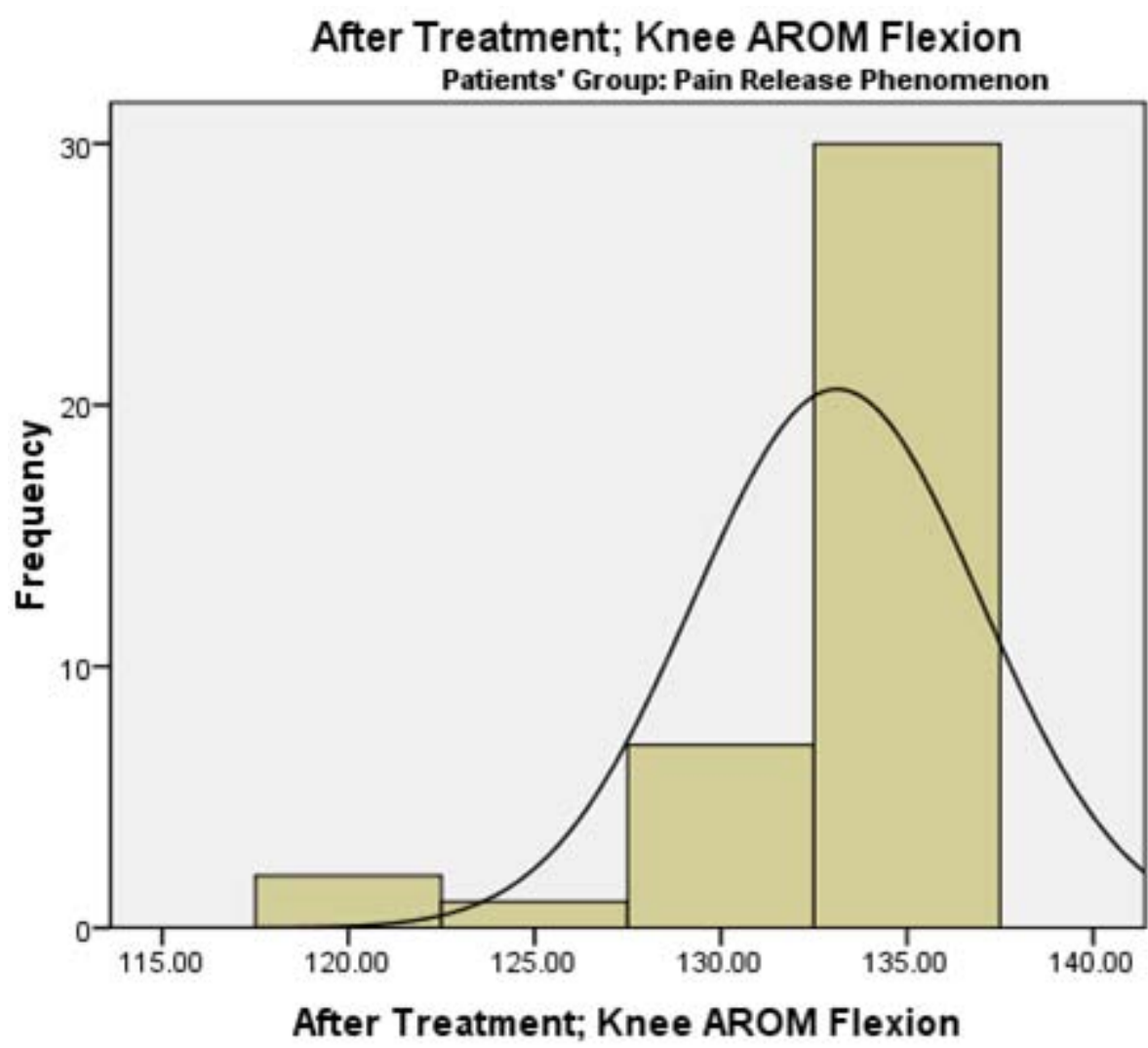

Mean $=133.12$

Std. Dev. $=3.871$ $\mathrm{N}=40$

Figure 16

For pain release phenomenon group, knee AROM flexion range before treatment found to be 13.12 on average with standard deviation 3.871, while curve is skewed positively.

Total Score: Functional Index Questionnaire

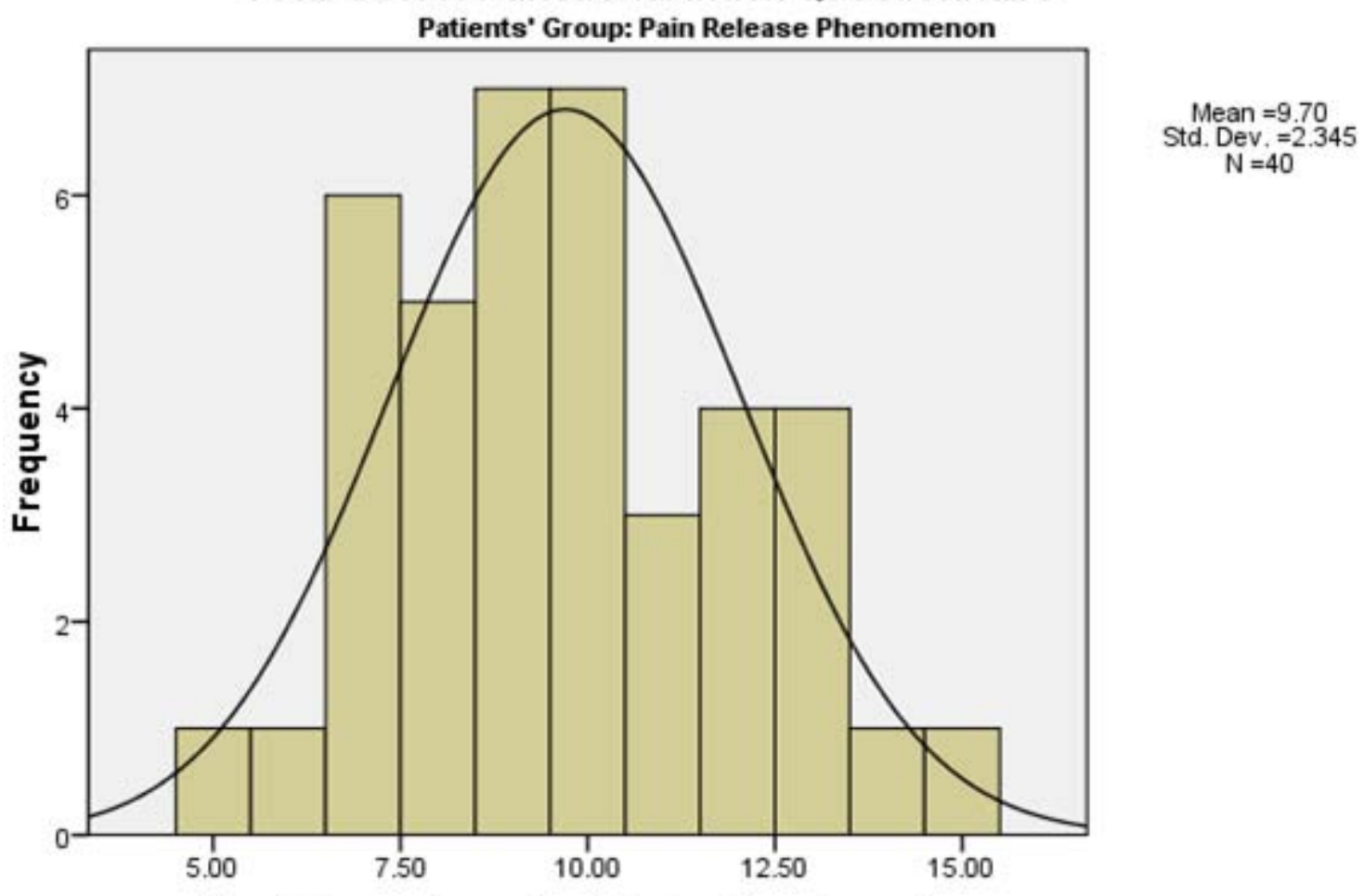

Total Score: Functional Index Questionnaire

Figure 17

For pain release phenomenon group, total score of Functional Index Questionnaire found to be 9.70 on average with standard deviation 2.345, while curve normally distributed.

Volume 5 Issue 7, July 2016 www.ijsr.net 


\section{Performance Level: Functional Index Questionnaire}

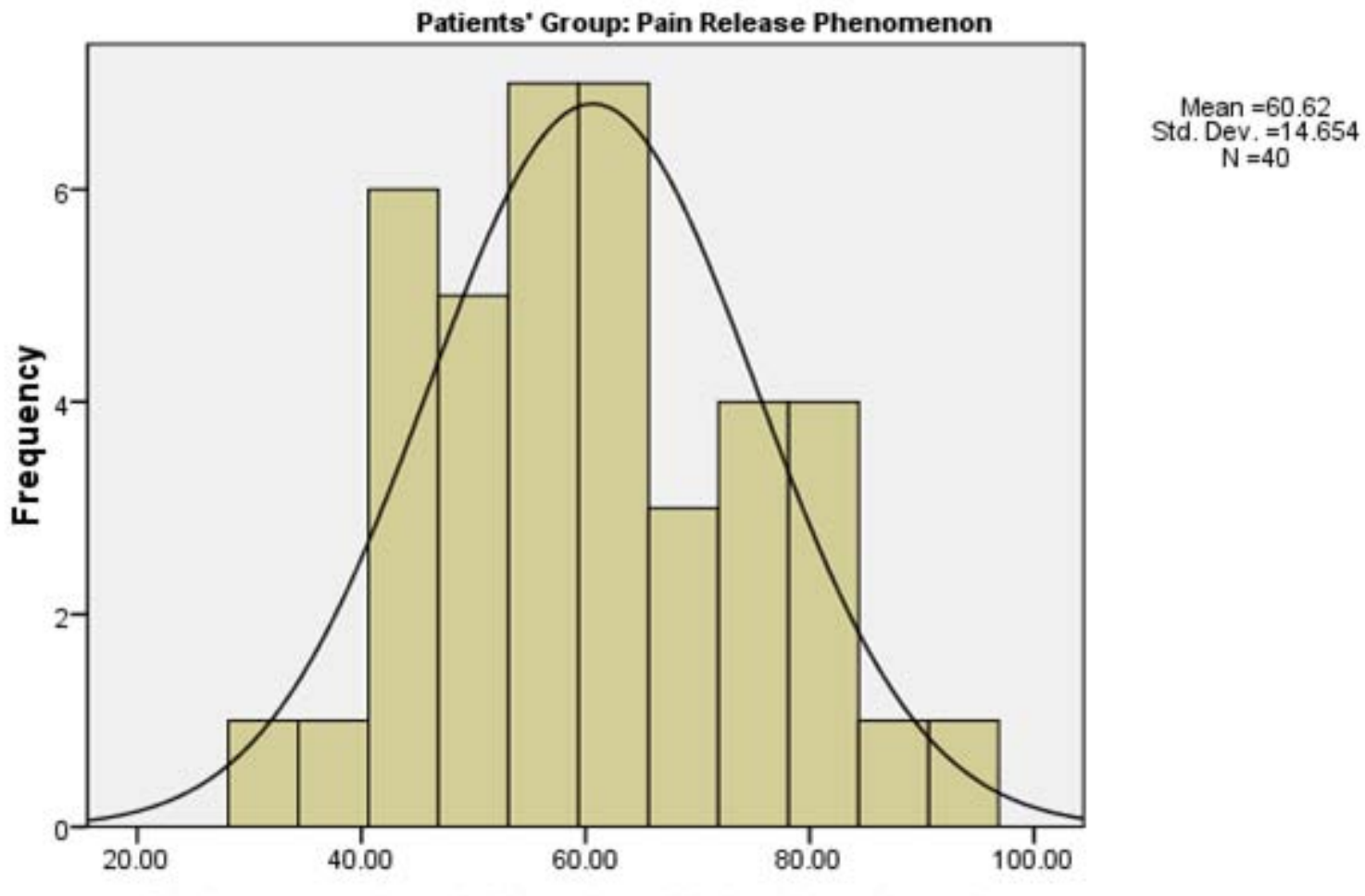

Performance Level: Functional Index Questionnaire

Figure 18

For pain release phenomenon group, performance level of Functional Index Questionnaire found to be 60.82 on average with standard deviation 14.654, while curve skewed positively towards higher values.

\section{Socioecnomic Status}

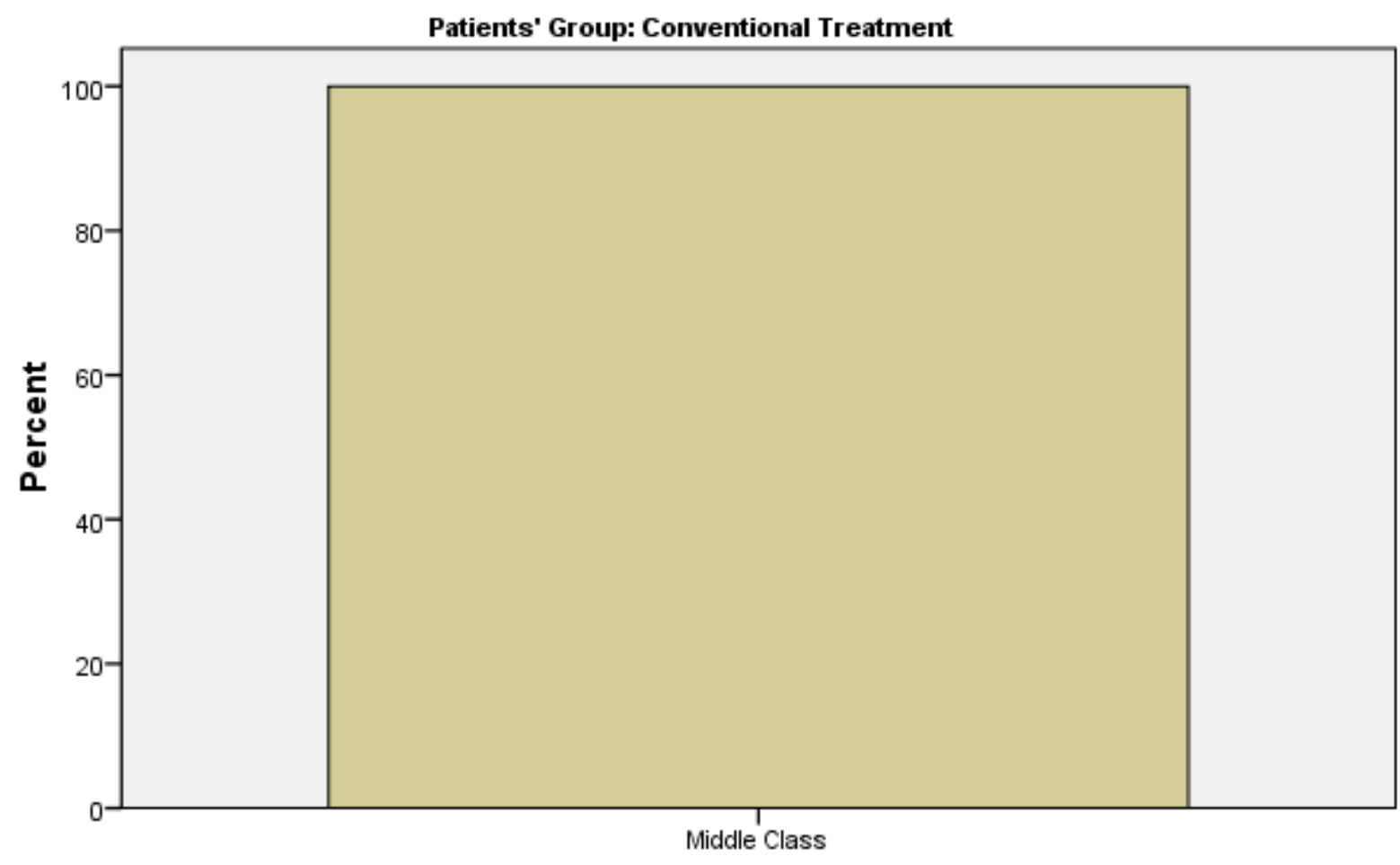

\section{Socioecnomic Status}

Figure 19

For conventional group all patients found belonging to middle class of socioeconomics.

Volume 5 Issue 7, July 2016

www.ijsr.net 


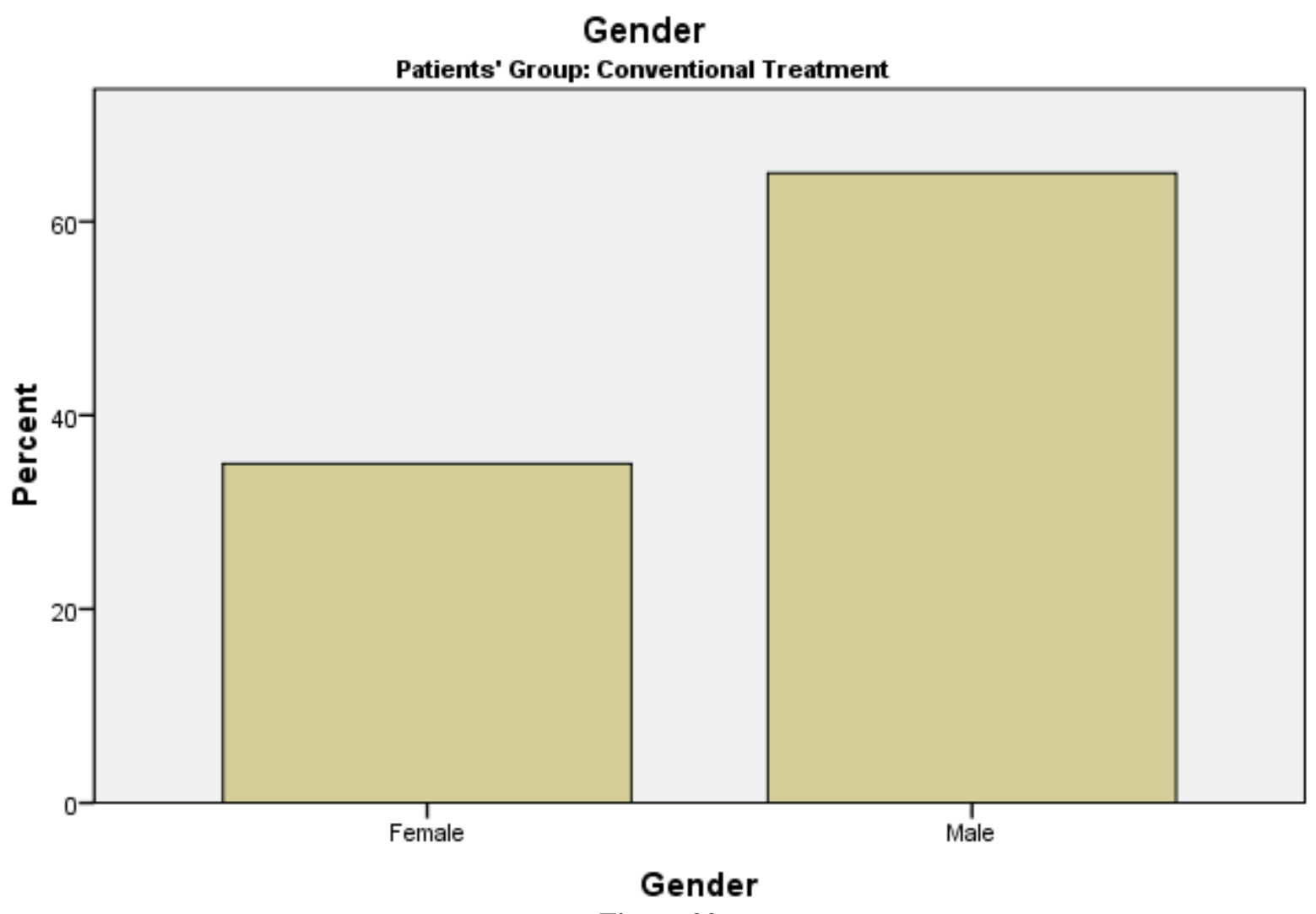

Figure 20

In conventional group, females were 14(35\%) and males were 26(65\%)

Knee Pain

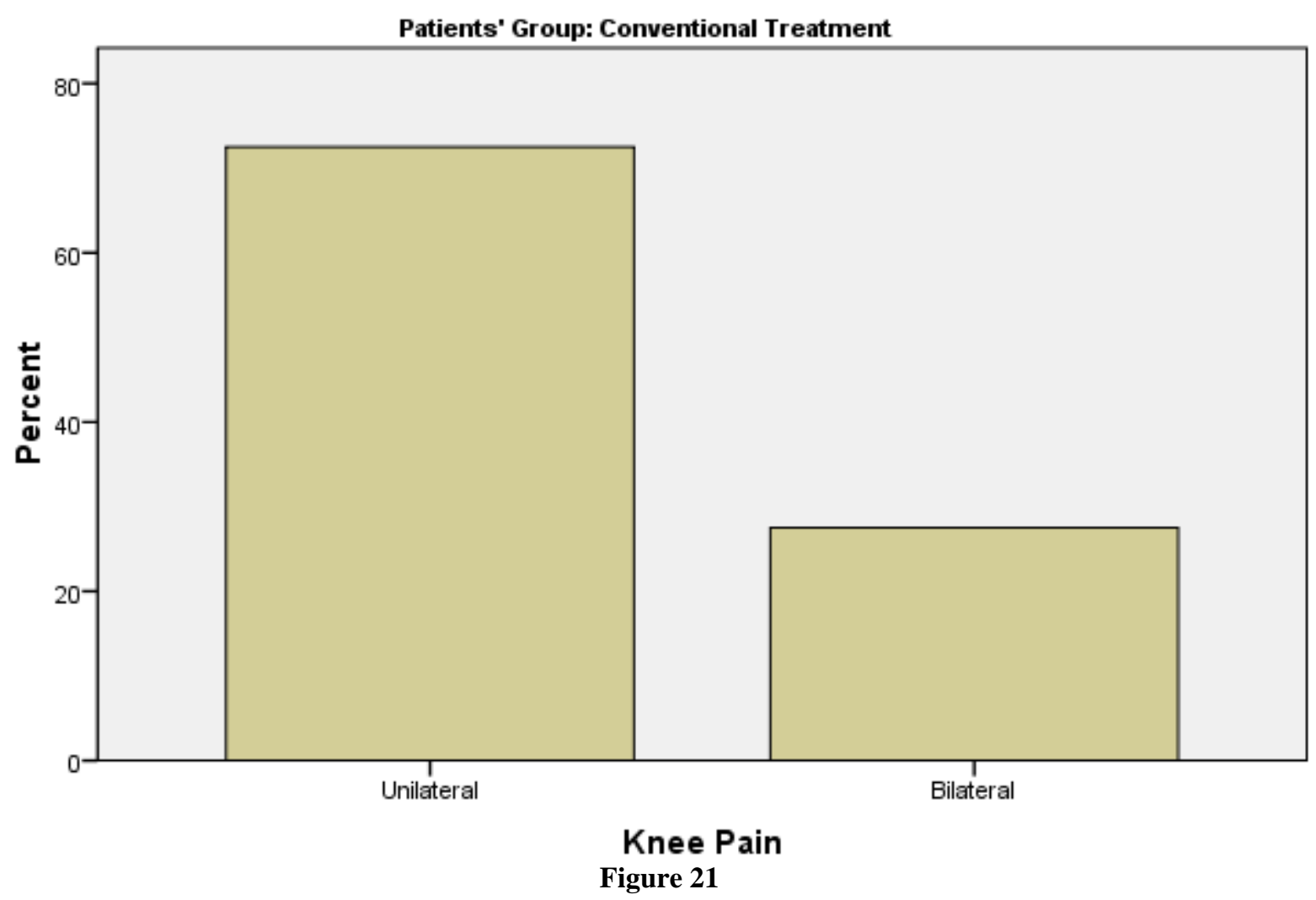

In conventional group, 29(72.5\%) were experiencing unilateral pain, while in 11(27.5\%) experiencing bilateral knee pain.

Volume 5 Issue 7, July 2016 www.ijsr.net

Licensed Under Creative Commons Attribution CC BY 


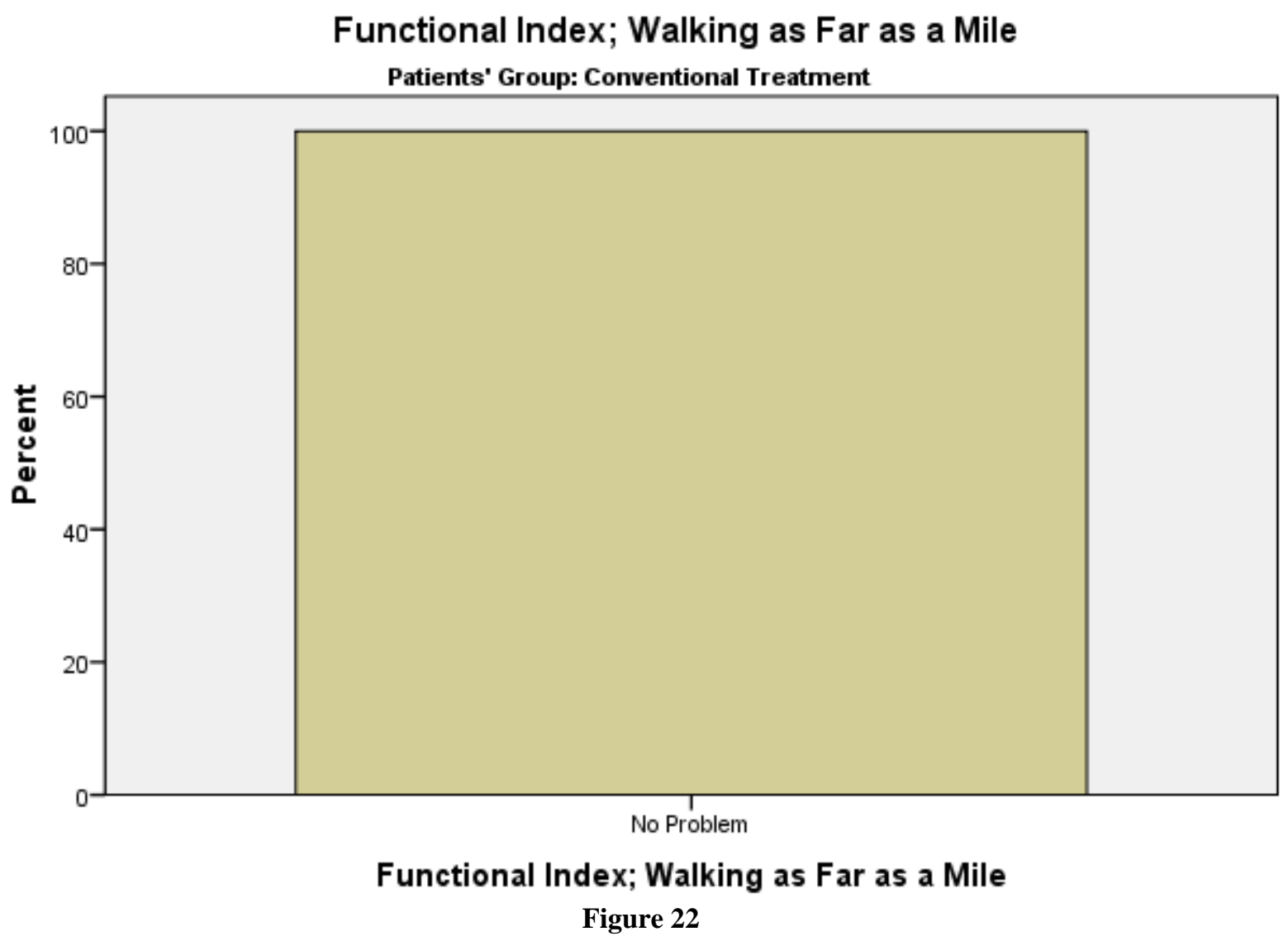

Conventional group all patients found with no problem while walking up to one mile

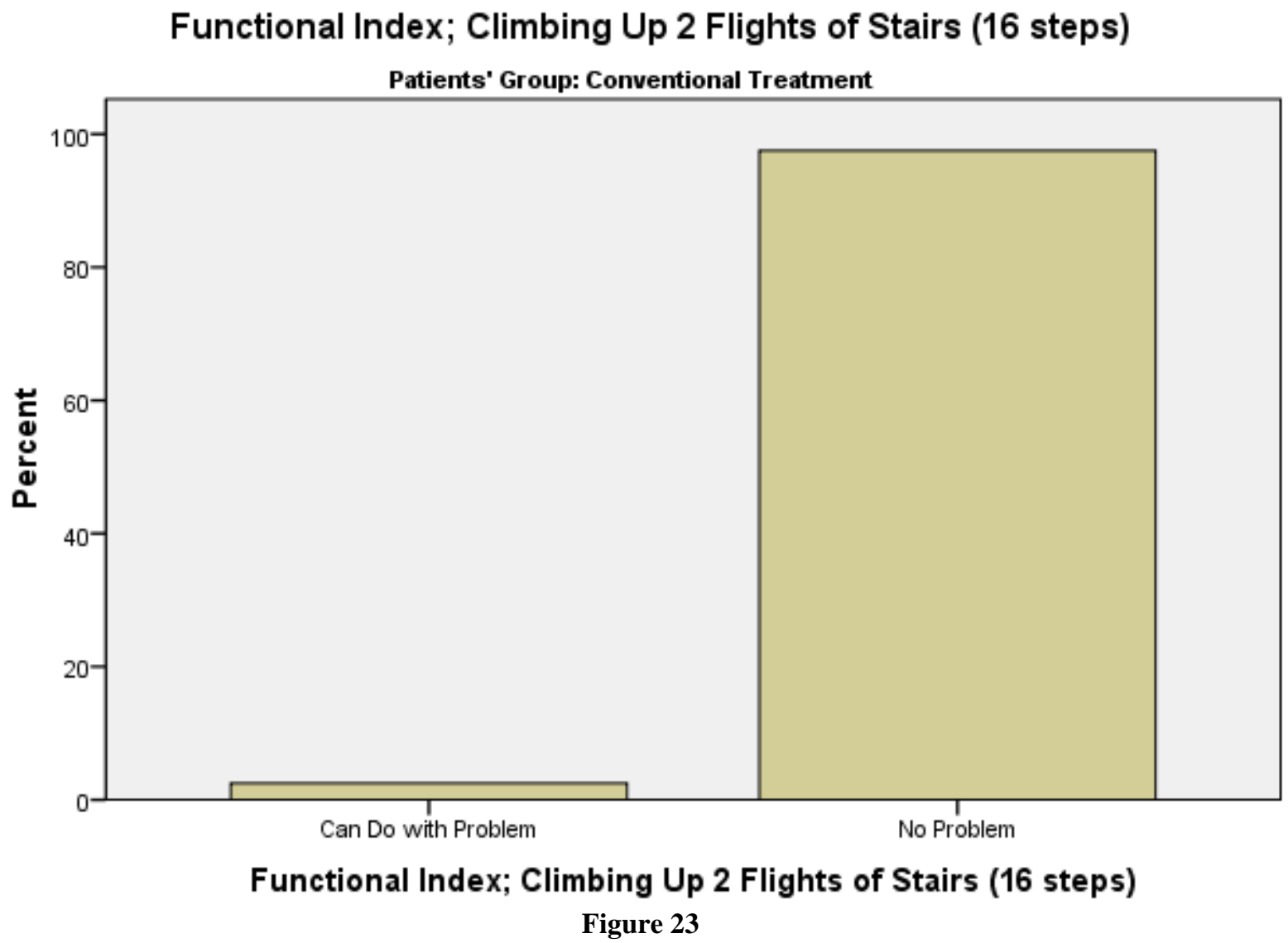

In conventional group, only $1(2.5 \%)$ found doing with problem while climbing up to two flights of stairs while rest of patients found with no problem.

Volume 5 Issue 7, July 2016 www.ijsr.net 
International Journal of Science and Research (IJSR)

ISSN (Online): 2319-7064

Index Copernicus Value (2013): 6.14 | Impact Factor (2015): 6.391

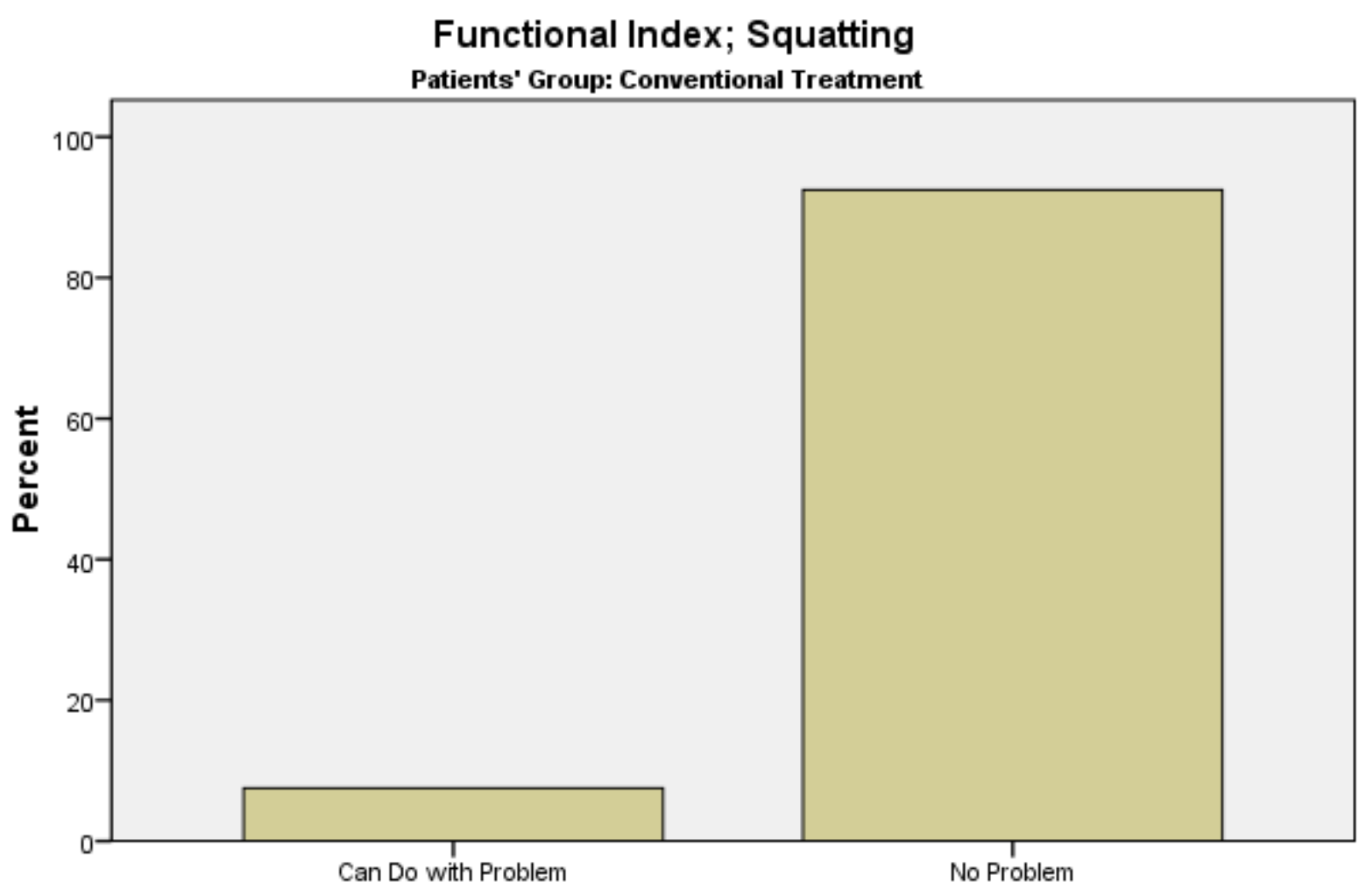

Functional Index; Squatting

Figure 24

In conventional group, 3(7.5\%) found doing squat with problem while rest of 37(92.5\%) found without problem.

Functional Index; Kneeling

Patients' Group: Conventional Treatment

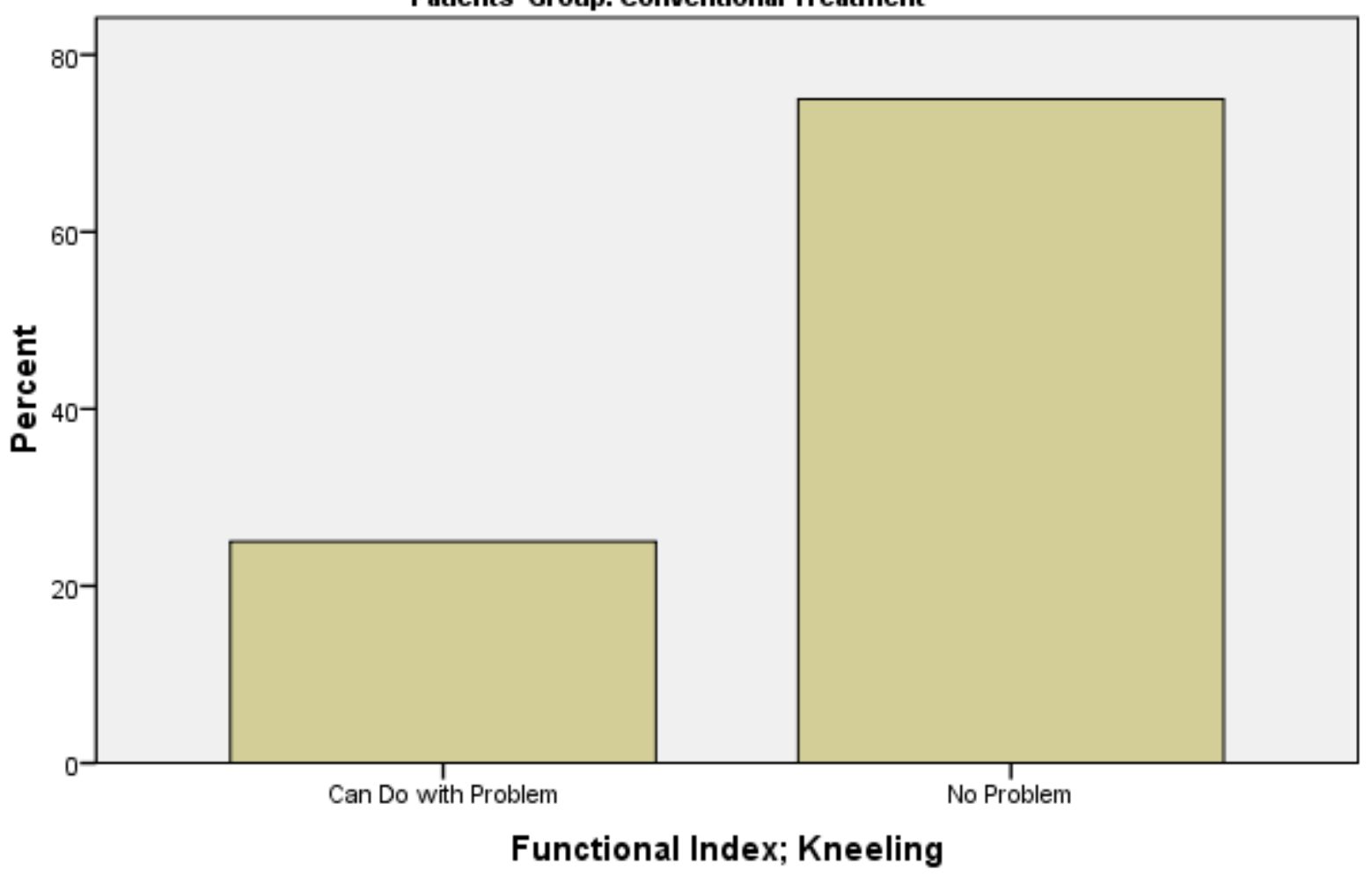

Figure 25

In conventional group, 10(25.0) patients had problem in kneeling, while 30(75\%) had no problem.

Volume 5 Issue 7, July 2016

www.ijsr.net

Licensed Under Creative Commons Attribution CC BY 


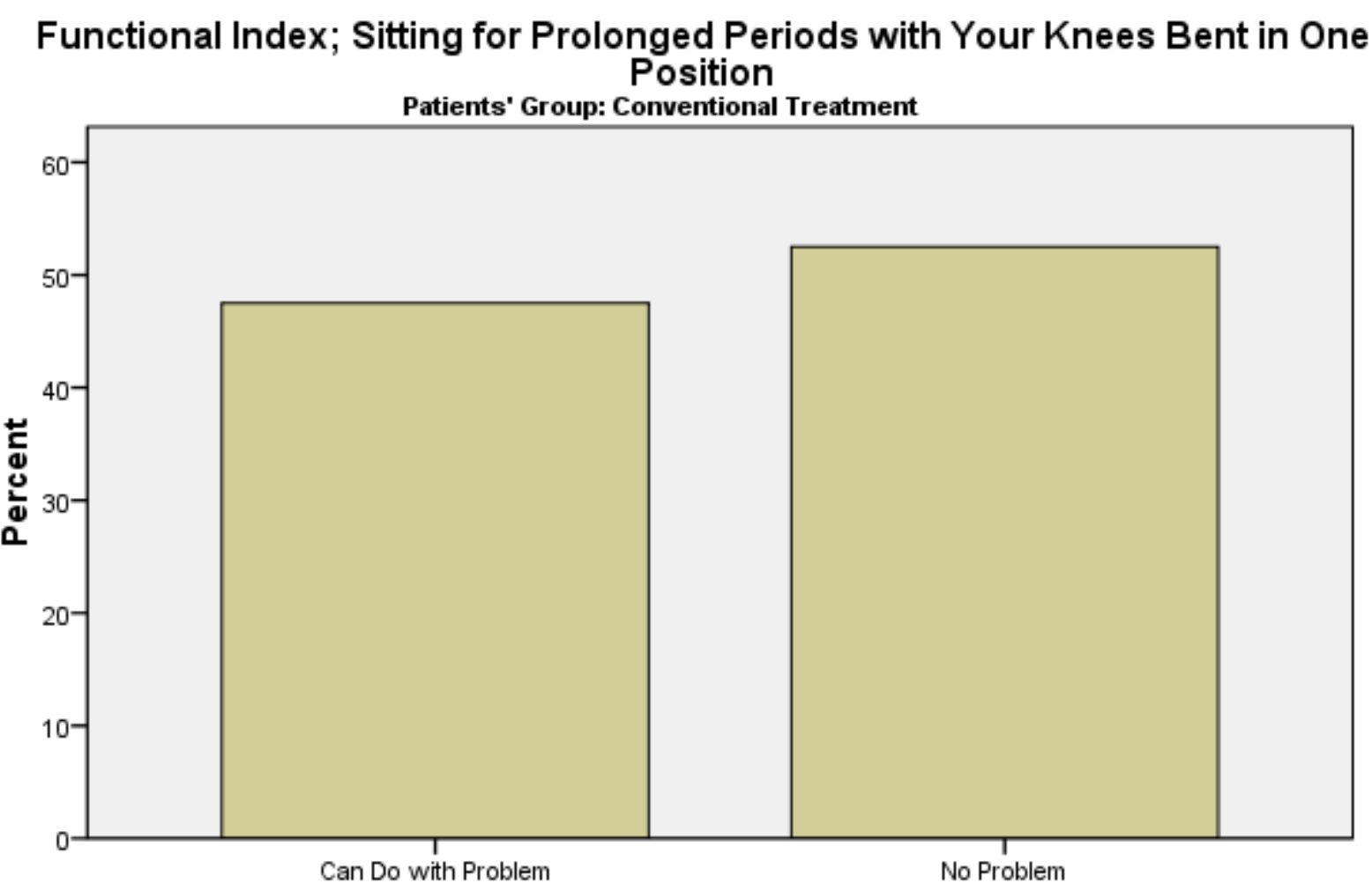

\section{Functional Index; Sitting for Prolonged Periods with Your Knees Bent in One Position \\ Figure 25}

In conventional group, sitting for prolonged periods with knees bent in one position, 19(47.5\%) found problematic while 21(52.5\%) found without problem.

\section{Functional Index; Climbing Up 4 Flights of Stairs (32 steps)}

Patients' Group: Conventional Treatment

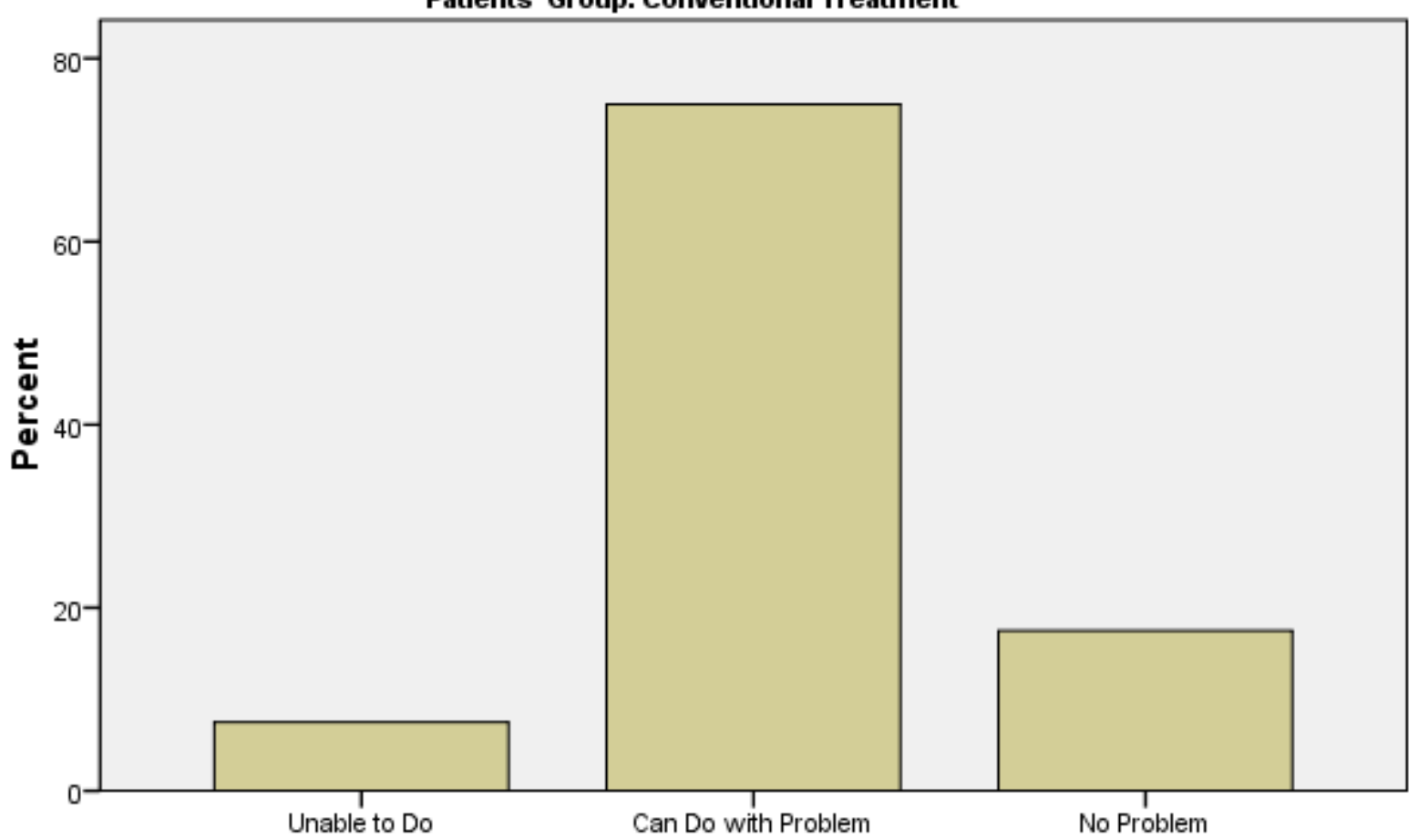

Functional Index; Climbing Up 4 Flights of Stairs (32 steps)

Figure 26

In conventional group, climbing up 4 flights of stairs, 3(7.5\%) found unable to do, 30(75\%) found with problem while 7(71.5\%) found without problem.

Volume 5 Issue 7, July 2016 www.ijsr.net 


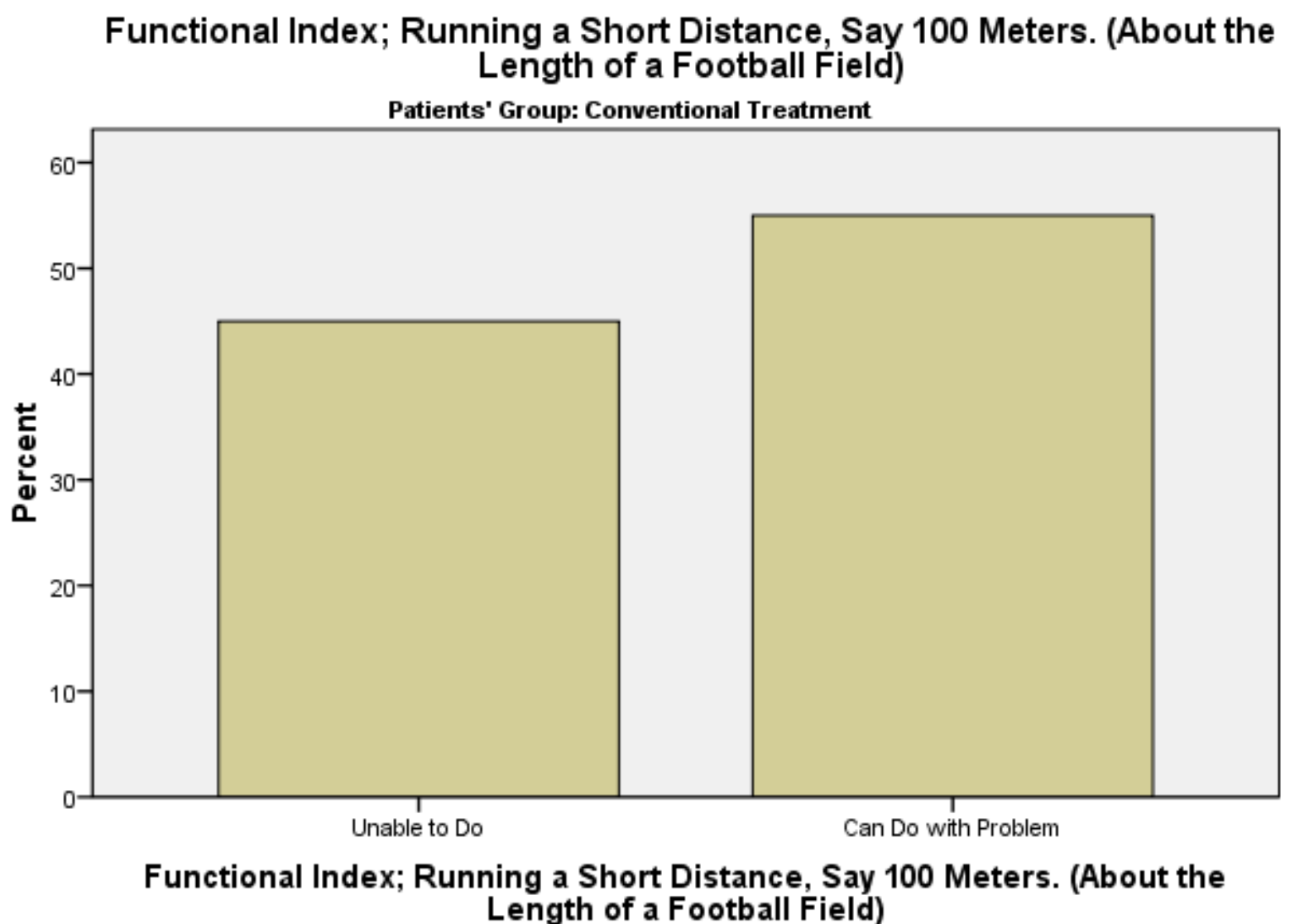

Figure 27

In conventional group, running a short distance up to 100 meters, $18(45 \%)$ were unable to do, 22(55\%) were doing with problem.

\section{Functional Index; Walking a Short Distance (About a City Block)}

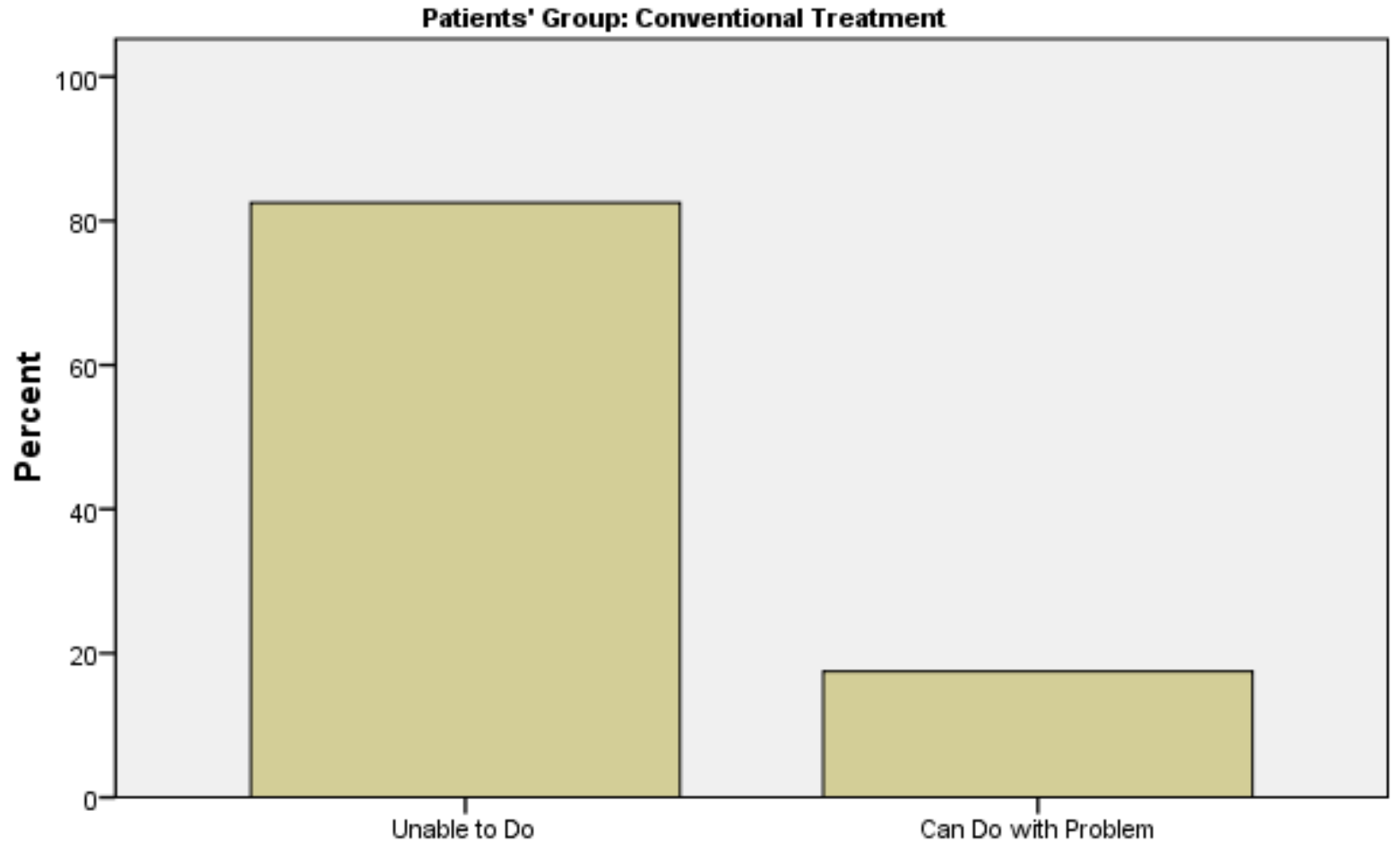

Functional Index; Walking a Short Distance (About a City Block)

Figure 28

In conventional group, walking a short distance, say a city block, 33(82.5\%) found unable to do while 7(17.5\%) found doing it with problem.

Volume 5 Issue 7, July 2016 www.ijsr.net 


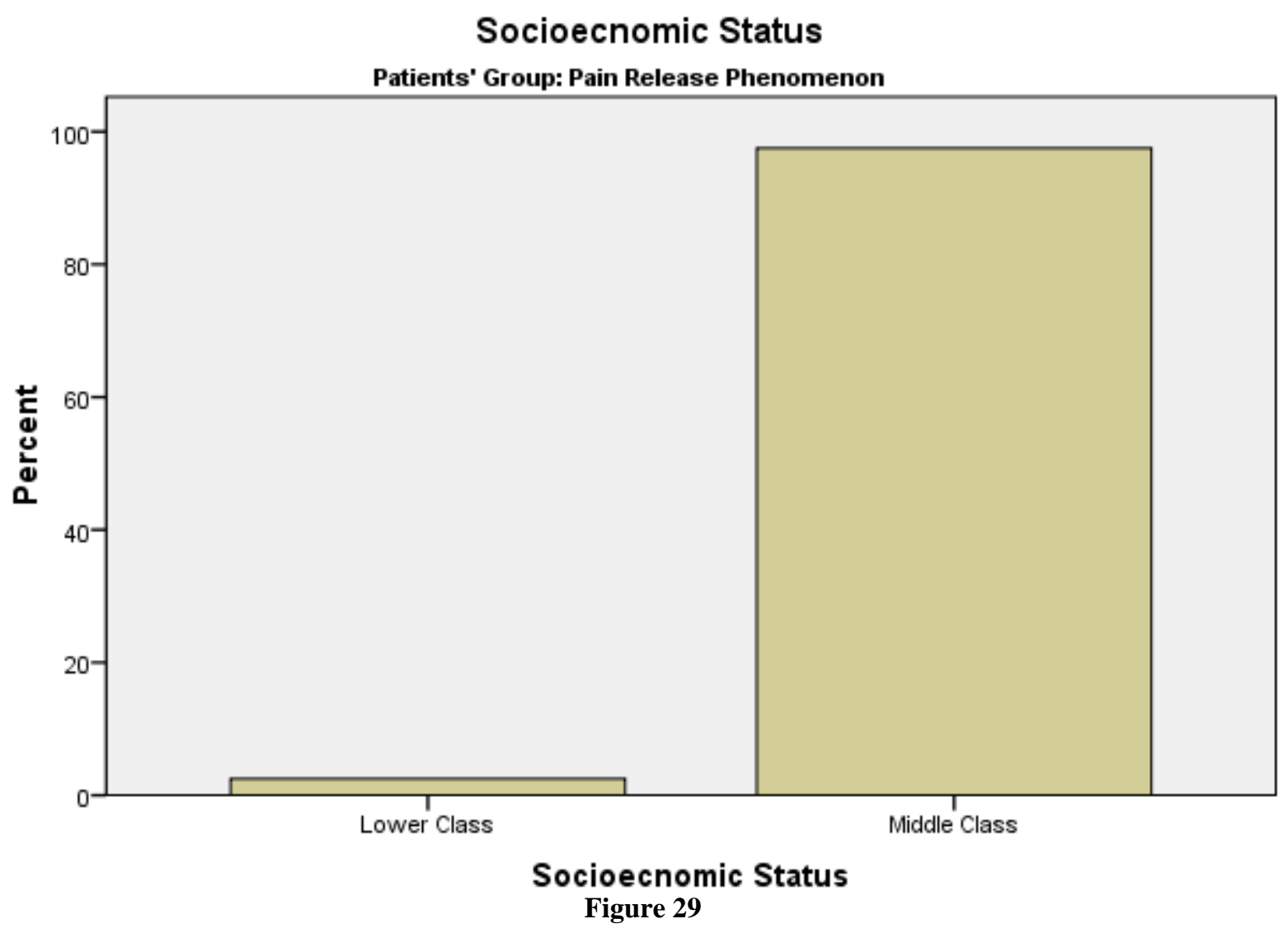

For pain release phenomenon group, $1(2.5 \%)$ patients were from lower class of socioeconomics while 39(97.5\%) belonging to middle class of socioeconomics.

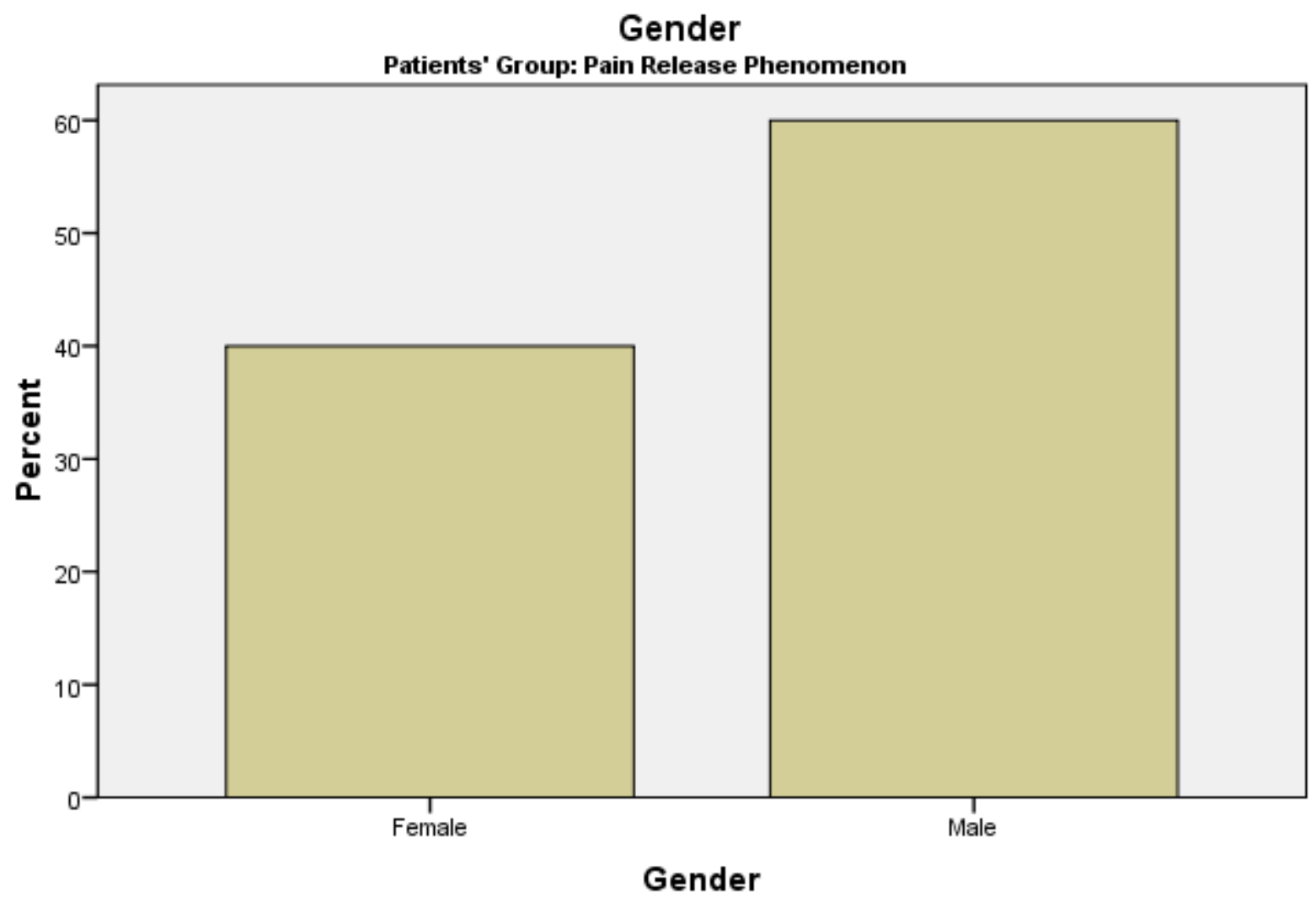

Figure 30

In pain release phenomenon group, females were 16(40\%) and males were 24(60\%)

Volume 5 Issue 7, July 2016 www.ijsr.net

Licensed Under Creative Commons Attribution CC BY 


\section{Knee Pain}

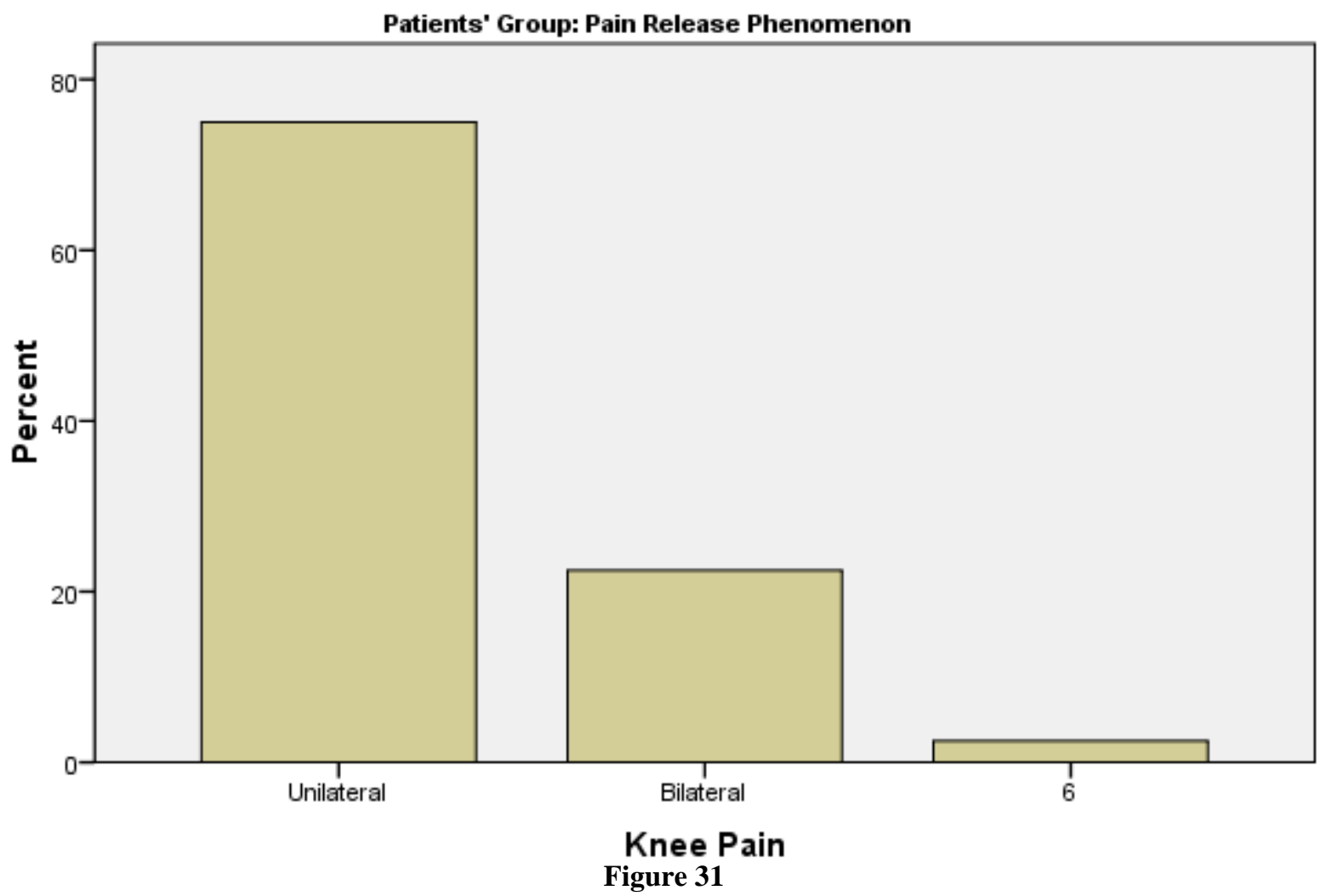

In pain release phenomenon group, 30(75\%) were experiencing unilateral pain, while in 9(22.5\%) experiencing bilateral knee pain.

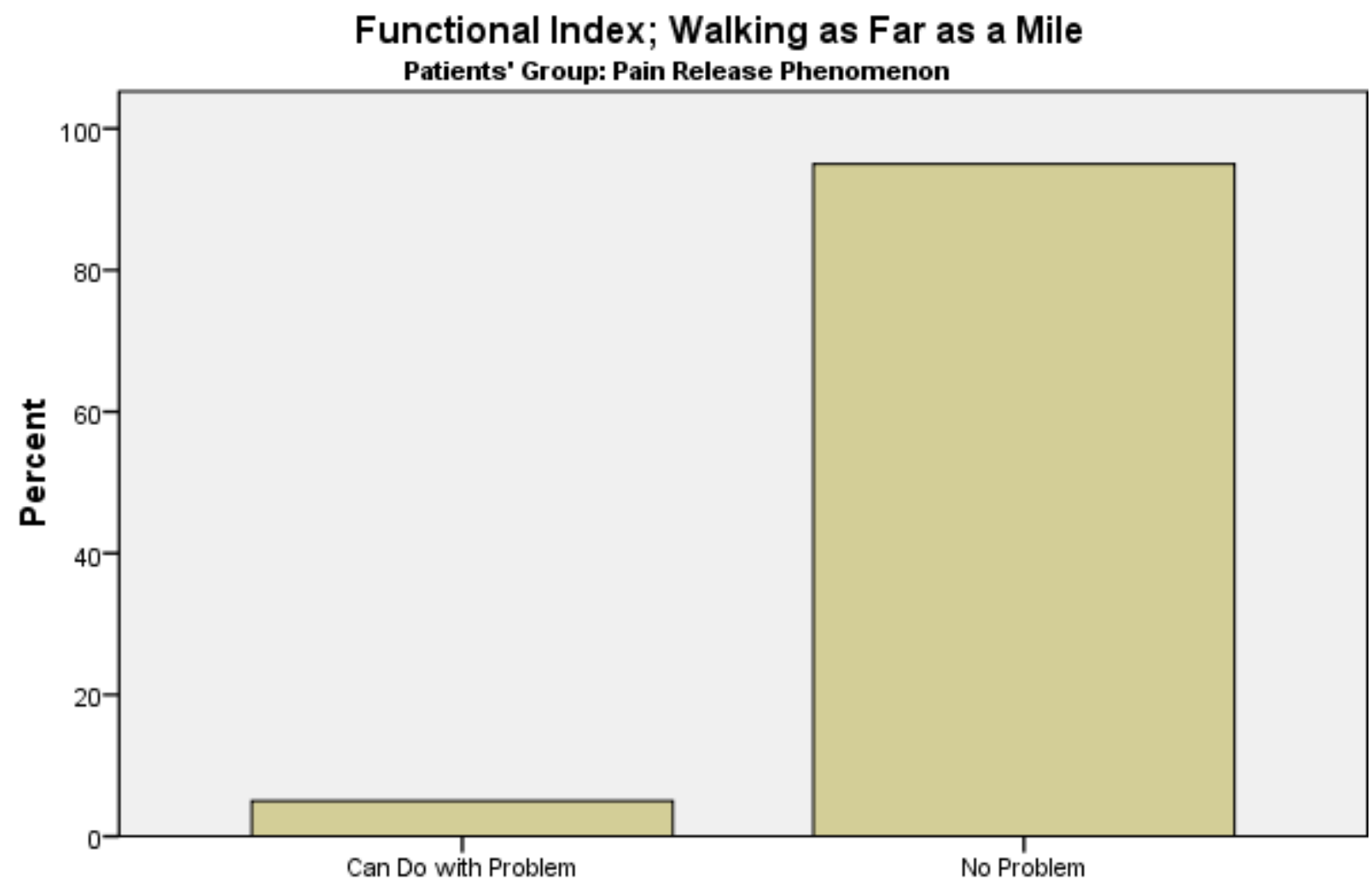

Functional Index; Walking as Far as a Mile

Figure 32

Pain release phenomenon group, 2(5\%) patients found walking a mile with problem while 38(95\%) found without problem.

Volume 5 Issue 7, July 2016 www.ijsr.net 
International Journal of Science and Research (IJSR)

ISSN (Online): 2319-7064

Index Copernicus Value (2013): 6.14 | Impact Factor (2015): 6.391

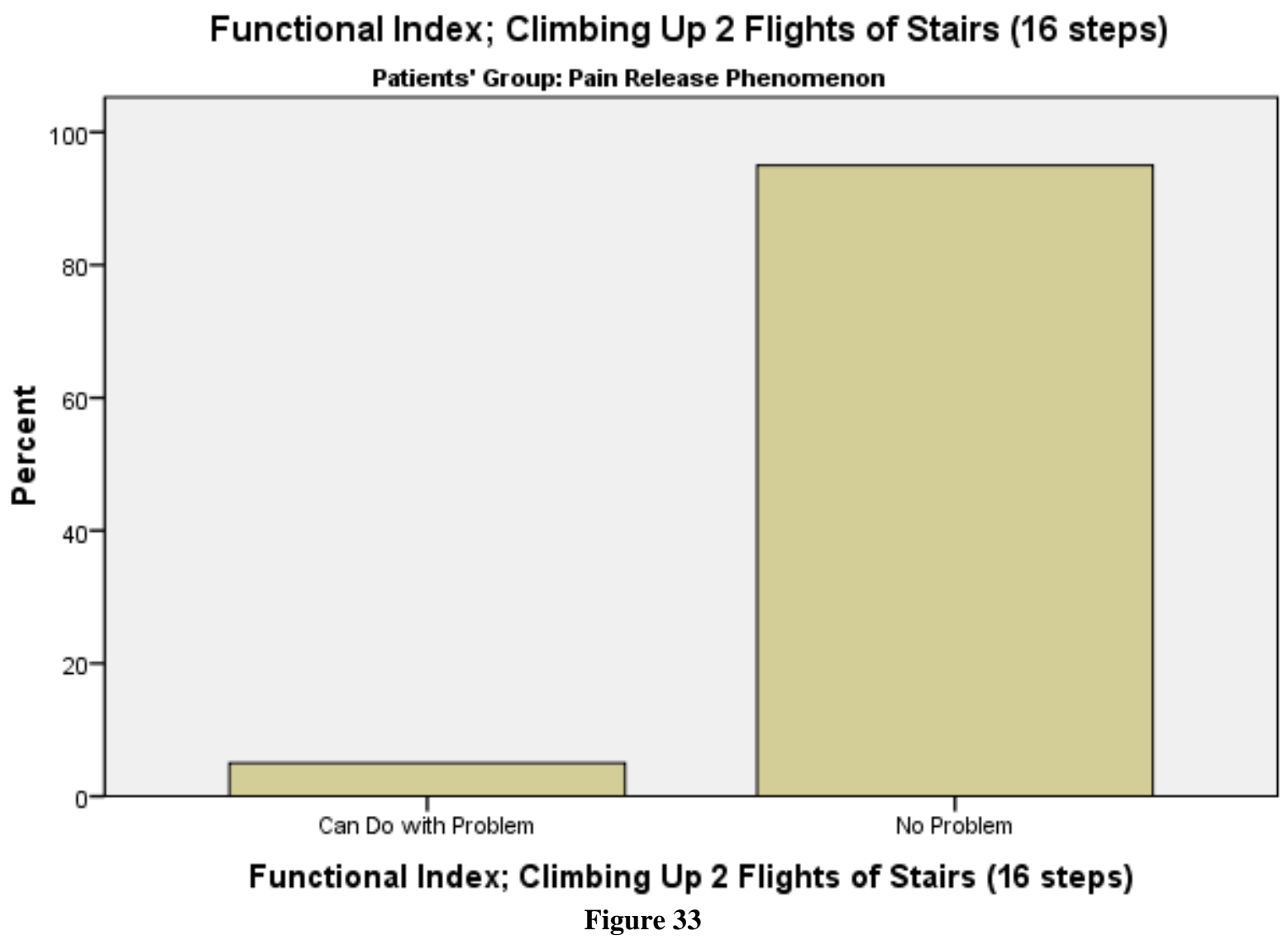

In pain release phenomenon group, only 2(5\%) found doing with problem while climbing up to two flights of stairs while

Functional Index; Squatting

Patients' Group: Pain Release Phenomenon

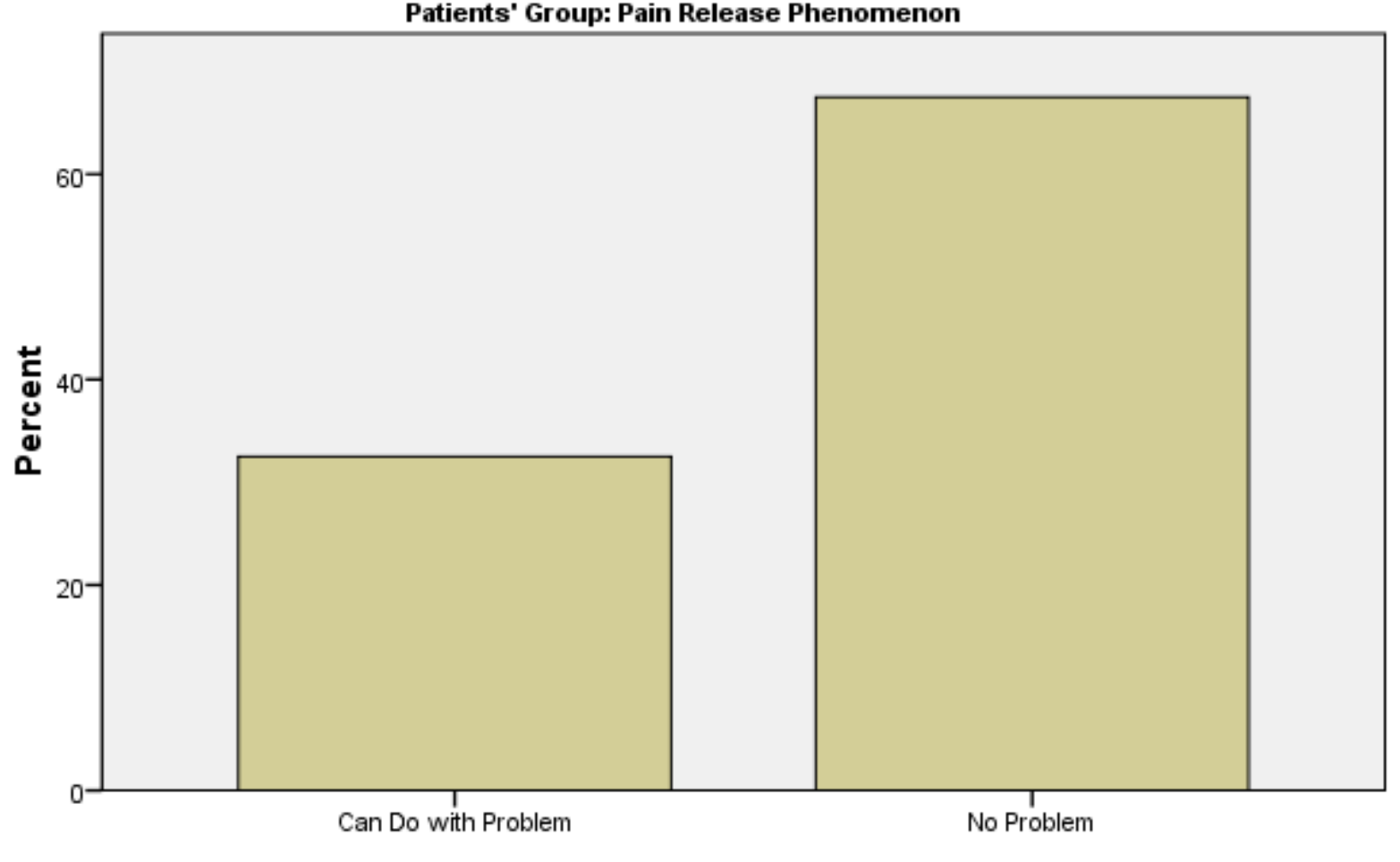

Functional Index; Squatting

Figure 34

In pain release phenomenon group, 13(32.5\%) found doing squat with problem while rest of 27(67.5\%) found without problem.

Volume 5 Issue 7, July 2016

www.ijsr.net

Licensed Under Creative Commons Attribution CC BY 


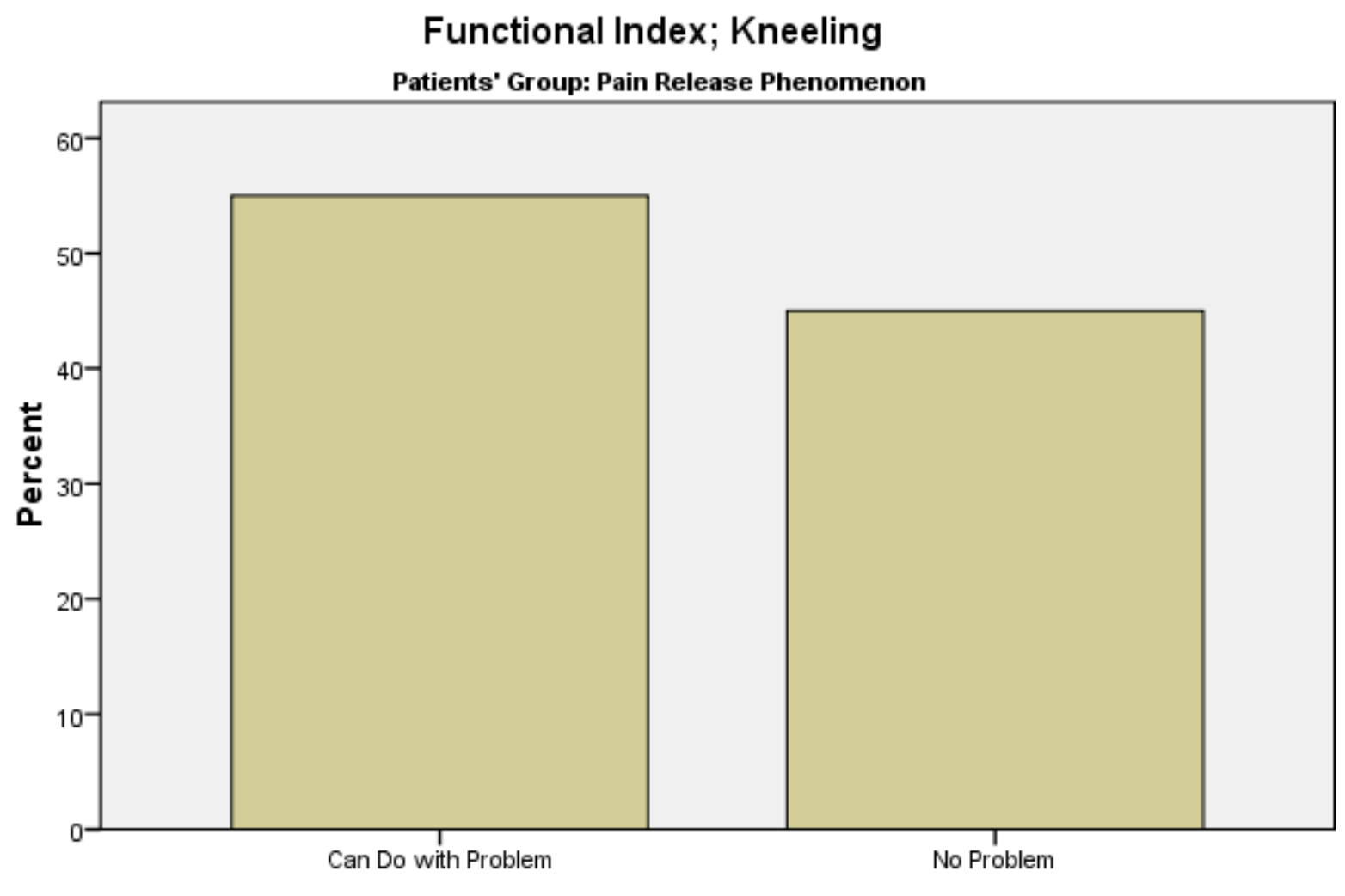

Functional Index; Kneeling

Figure 35

In pain release phenomenon group, 22(55.0) patients had problem in kneeling, while 18(45\%) had no problem.

Functional Index; Sitting for Prolonged Periods with Your Knees Bent in One Position

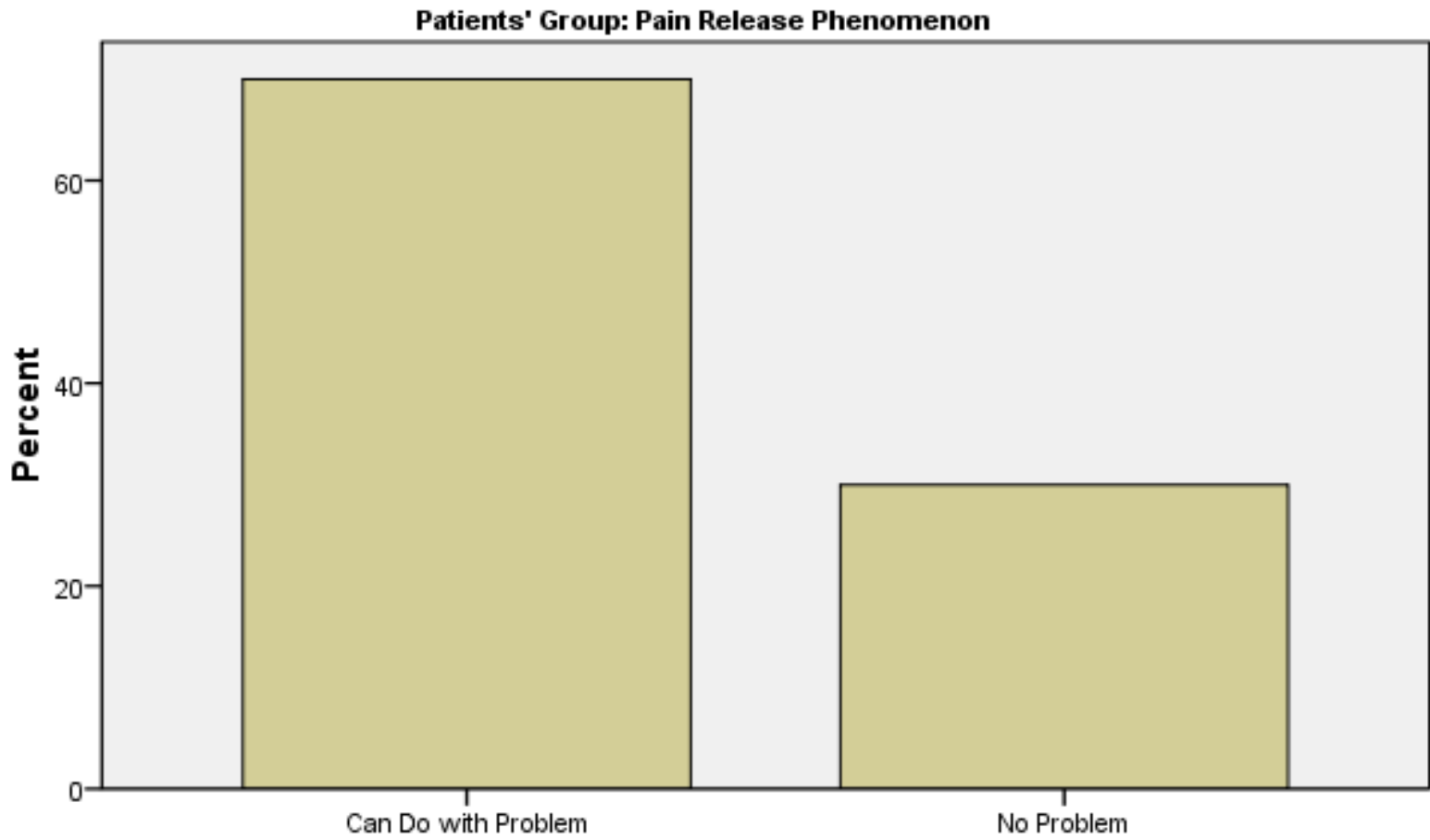

Functional Index; Sitting for Prolonged Periods with Your Knees Bent in One Position

Figure 36

In pain release phenomenon group, sitting for prolonged periods with knees bent in one position, 28(70\%) found problematic while 12(30\%) found without problem.

Volume 5 Issue 7, July 2016

www.ijsr.net

Licensed Under Creative Commons Attribution CC BY 


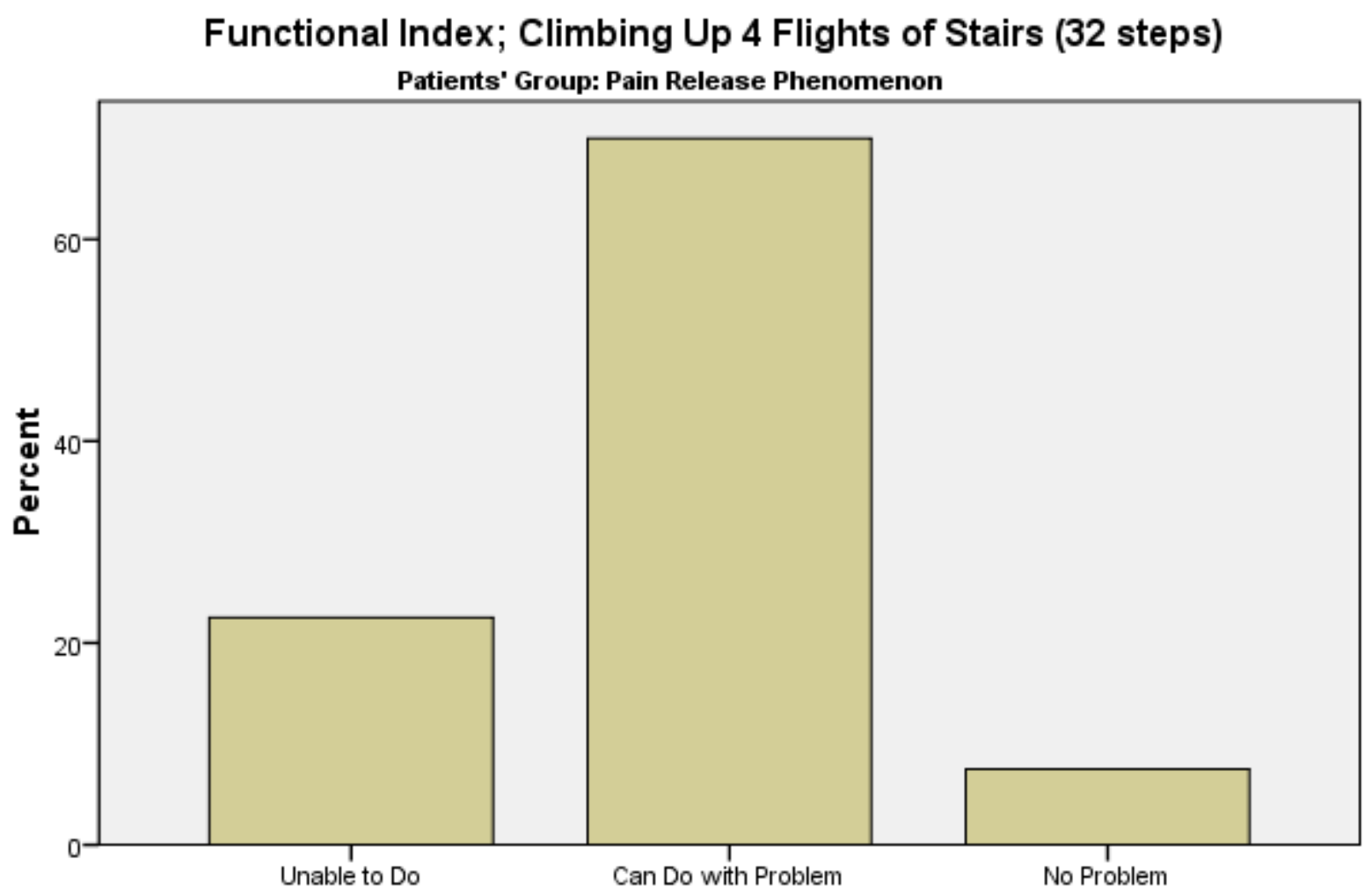

Functional Index; Climbing Up 4 Flights of Stairs (32 steps)

Figure 37

In pain release phenomenon group, climbing up 4 flights of stairs, $4(22.5 \%)$ found unable to do, 28(70\%) found with problem while 3(7.5\%) found without problem.

Functional Index; Running a Short Distance, Say 100 Meters. (About the Length of a Football Field)

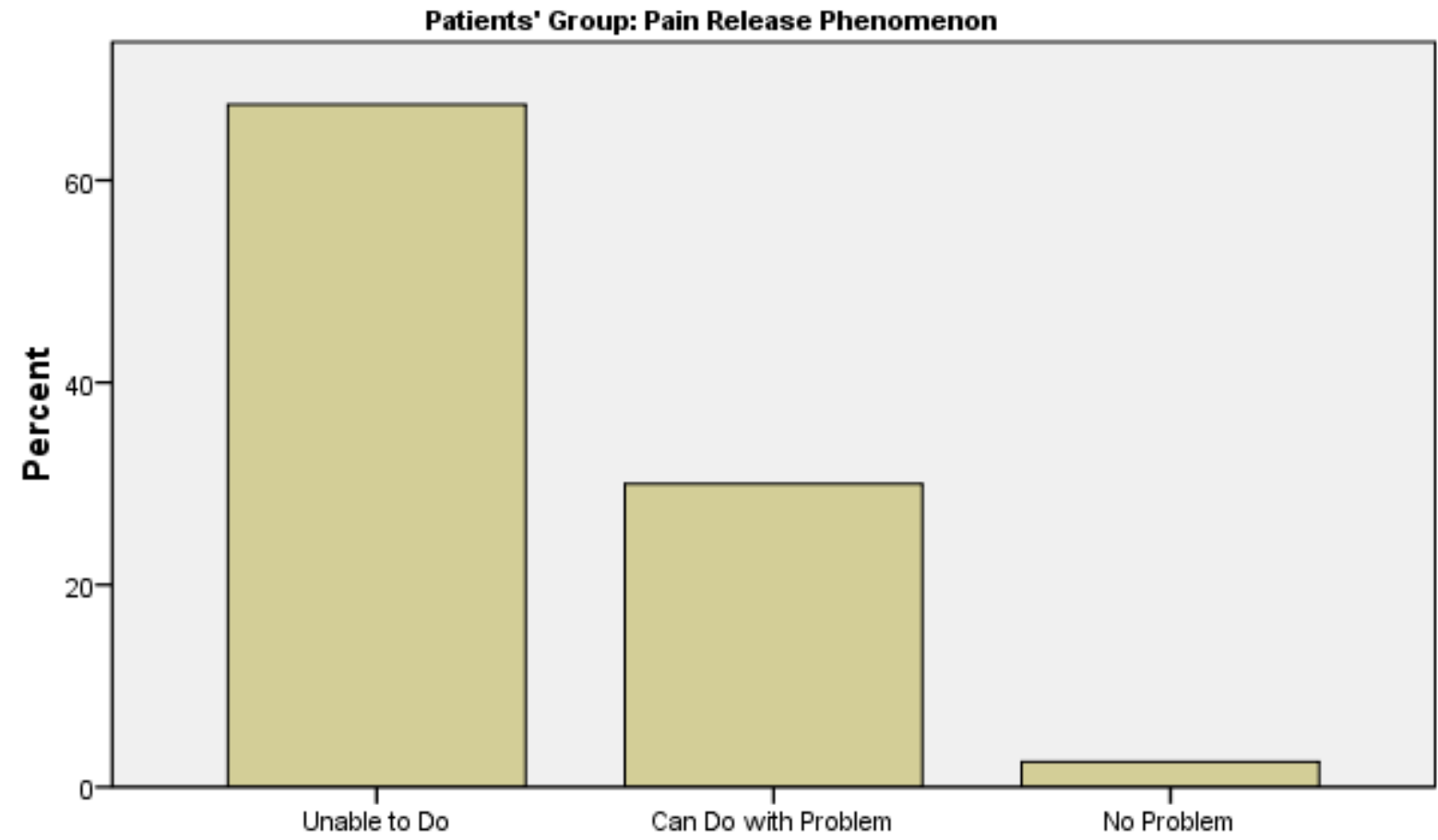

Functional Index; Running a Short Distance, Say 100 Meters. (About the Length of a Football Field)

Figure 38

In pain release phenomenon group, running a short distance up to 100 meters, 27(67.5\%) were unable to do, 12(30\%) were

Volume 5 Issue 7, July 2016 www.ijsr.net

Licensed Under Creative Commons Attribution CC BY 


\section{Functional Index; Walking a Short Distance (About a City Block)}

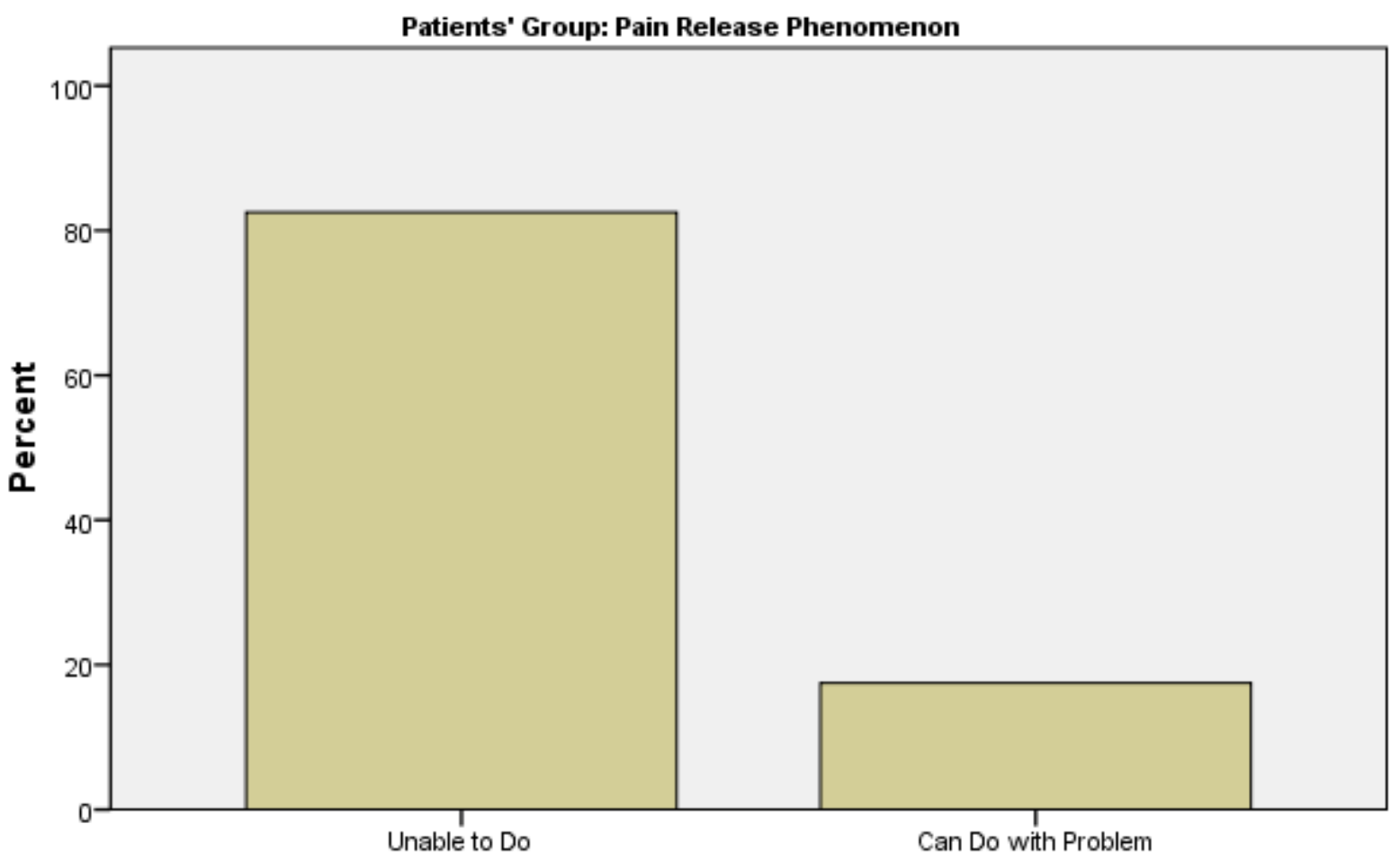

Functional Index; Walking a Short Distance (About a City Block)

Figure 39

In pain release phenomenon group, walking a short distance, say a city block, 33(82.5\%) found unable to do while $7(17.5 \%)$ found doing it with problem.

\section{Discussion}

Despite the availability of a number of treatment options for PFPS there exist fewer consensuses on the effectiveness of a specific set of exercises or treatment technique. More work has been done regarding the biomechanical causative factors of PFPS rather than intervention based studies. In current setting this study would help adding to literature regarding interventions for PFPS.

Routine physical therapy treatment for PFPS has been in practice since many years now. There is a need for innovation new treatment approaches in order to improve functional status and improve quality of life of the patients.

It has been proven that exercise has strong pain-minimizing effects, however which specific exercise therapy to use is still questionable.(14) Current study was aimed at testing the effectiveness of an innovative technique combined with the conventional physical therapy practices.

The pain release phenomenon (PRP) (developed by Brian Mulligan) is a relatively new treatment approach. This study has employed and tested this treatment approach for the patients of patellofemoral pain syndrome. Our study has shown markedly improved pain and functional status in six weeks among the group receiving the pain release phenomenon (PRP). The improvement in Pain and functional status in Group B who received both PRP and traditional treatment was much evident as compared to the group receiving standardized/conventional treatment only. Effect size was also larger for both VAS and Functional index questionnaire.

The results of this study are supported by another randomized controlled trial that has concluded that a sixweek, six session physical therapy regimen improves the level of function and reduces the level of pain in patients of patellofemoral pain syndrome.(15) The difference however lies in the frequency of physical therapy sessions. Which were greater in our study i.e. thrice per week. Quadriceps strengthening has long been use in improving function and pain in PFPS and literature has shown a strong evidence in support of the use of these exercises either alone or in conjunction with other interventions. They have their benefits.(16) These suggestions and findings are consistent with the results of our study. Group (A) received only conventional treatment and that did include quadriceps strengthening focusing on VMO. Group B receive both PRP and conventional treatment. The functional status was improved in both groups and pain was also reduced. However, the differences were more marked for Group B.

\section{Conclusion}

This study concludes that Pain release phenomenon is an effective technique in reducing pain and improving function of knee in patients with patellofemoral pain when combined with conventional treatment and home exercise plan over a period of six weeks. 


\section{International Journal of Science and Research (IJSR) \\ ISSN (Online): 2319-7064 \\ Index Copernicus Value (2013): 6.14 | Impact Factor (2015): 6.391}

\section{Dedication}

Dedicated to my beloved parents, there is no one more affectionate and loving than my parents on whole face of earth

\section{Acknowledgement}

First of all I bow before Allah Almighty, omnipresent, the most merciful, the most compassionate, the most gracious and beneficent whose help and guidance is always solicited at every step and at every moment of my life.

I am greatly indebted to Dr. Ashfaq Ahamd, Head of Department, University Institute of University of Lahore, for his support in promoting my research project, his vigilant surveillance and administrative help in overcoming the challenges in completing my work.

I am also indebted to Dr Umair Ahmad, his patience, guidance and moral support, considerably beyond the call of duty and enabling me to complete the project in timely manner. Again I am thankful to my supervisor, Dr Ashfaq Ahmad for his tireless efforts, professional expertise, guidance, advice and support, without which this work would have not been possible.

In the end I would like to acknowledge the support all patients who participated in this survey to achieve this goal. Every accompalishment in my life in the end is possible due to support and trust of my family.

\section{References}

[1] Biber R, Gregory A. Overuse injuries in youth sports: is there such a thing as too much sports? Pediatric Annals. 2010;39(5):286.

[2] Samuels ML, Witmer JA, Schaffner A. Statistics for the life sciences: Pearson Education; 2012.

[3] Boling M, Padua D, Marshall S, Guskiewicz K, Pyne S, Beutler A. Gender differences in the incidence and prevalence of patellofemoral pain syndrome. Scandinavian Journal Of Medicine \& Science In Sports. 2010;20(5):725-30.

[4] Ferber R, Kendall KD, Farr L. Changes in knee biomechanics after a hip-abductor strengthening protocol for runners with patellofemoral pain syndrome. Journal Of Athletic Training. 2011;46(2):142.

[5] Myer GD, Ford KR, Foss KDB, Goodman A, Ceasar A, Rauh MJ, et al. The incidence and potential pathomechanics of patellofemoral pain in female athletes. Clinical Biomechanics. 2010;25(7):700-7.

[6] McDowell JM, Johnson GM, Hetherington BH. Mulligan Concept manual therapy: Standardizing annotation. Manual Therapy. 2014;19(5):499-503.

[7] Fukuda TY, Rossetto FM, MAGALHãES E, Bryk FF, Garcia Lucareli PR, de Almeida Carvalho NA. Shortterm effects of hip abductors and lateral rotators strengthening in females with patellofemoral pain syndrome: a randomized controlled clinical trial. Journal of Orthopaedic \& Sports Physical Therapy. 2010;40(11):736-42.

[8] Bolgla LA, Boling MC. An update for the conservative management of patellofemoral pain syndrome: a systematic review of the literature from 2000 to 2010 . International Journal Of Sports Physical Therapy. 2011;6(2):112.

[9] Davis IS, Powers C. Patellofemoral Pain Syndrome: Proximal, Distal, and Local Factors-International Research Retreat, April 30-May 2, 2009, Baltimore, Maryland. Journal of Orthopaedic \& Sports Physical Therapy. 2010;40(3):A1-A48.

[10] Foss KDB, Myer GD, Chen SS, Hewett TE. Expected prevalence from the differential diagnosis of anterior knee pain in adolescent female athletes during preparticipation screening. Journal of Athletic Training. 2012;47(5):519-24.

[11]Dy C, Franco N, Ma Y, Mazumdar M, McCarthy M, Della Valle AG. Complications after patello-femoral versus total knee replacement in the treatment of isolated patello-femoral osteoarthritis. A meta-analysis. Knee Surgery, Sports Traumatology, Arthroscopy. 2012;20(11):2174-90.

[12] Khayambashi K, Mohammadkhani Z, Ghaznavi K, Lyle MA, Powers CM. The effects of isolated hip abductor and external rotator muscle strengthening on pain, health status, and hip strength in females with patellofemoral pain: a randomized controlled trial. Journal of Orthopaedic \& Sports Physical Therapy. 2012;42(1):22-9.

[13] Nakagawa TH, Moriya ÉT, Maciel CD, Serrão FV. Trunk, pelvis, hip, and knee kinematics, hip strength, and gluteal muscle activation during a single-leg squat in males and females with and without patellofemoral pain syndrome. Journal of Orthopaedic \& Sports Physical Therapy. 2012;42(6):491-501.

[14] Lee S-P, Souza RB, Powers CM. The influence of hip abductor muscle performance on dynamic postural stability in females with patellofemoral pain. Gait \& Posture. 2012;36(3):425-9.

[15] Dy C, Franco N, Ma Y, Mazumdar M, McCarthy M, Della Valle AG. Complications after patello-femoral versus total knee replacement in the treatment of isolated patello-femoral osteoarthritis. A meta-analysis. Knee Surgery, Sports Traumatology, Arthroscopy. 2012;20(11):2174-90. 


\section{Proforma}

Serial no:

Date:

Age (Years):

Occupation:

Cell no:

Weight (kg):

Socioeconomic Status

$\square$ Lower Class $\square$ Middle Class $\square$ Upper Class

Gender

$\square$ Male $\square$ Female

Knee Pain:

$\square$ Unilateral $\square$ Bilateral

\section{Visual Analogue Pain Scale}

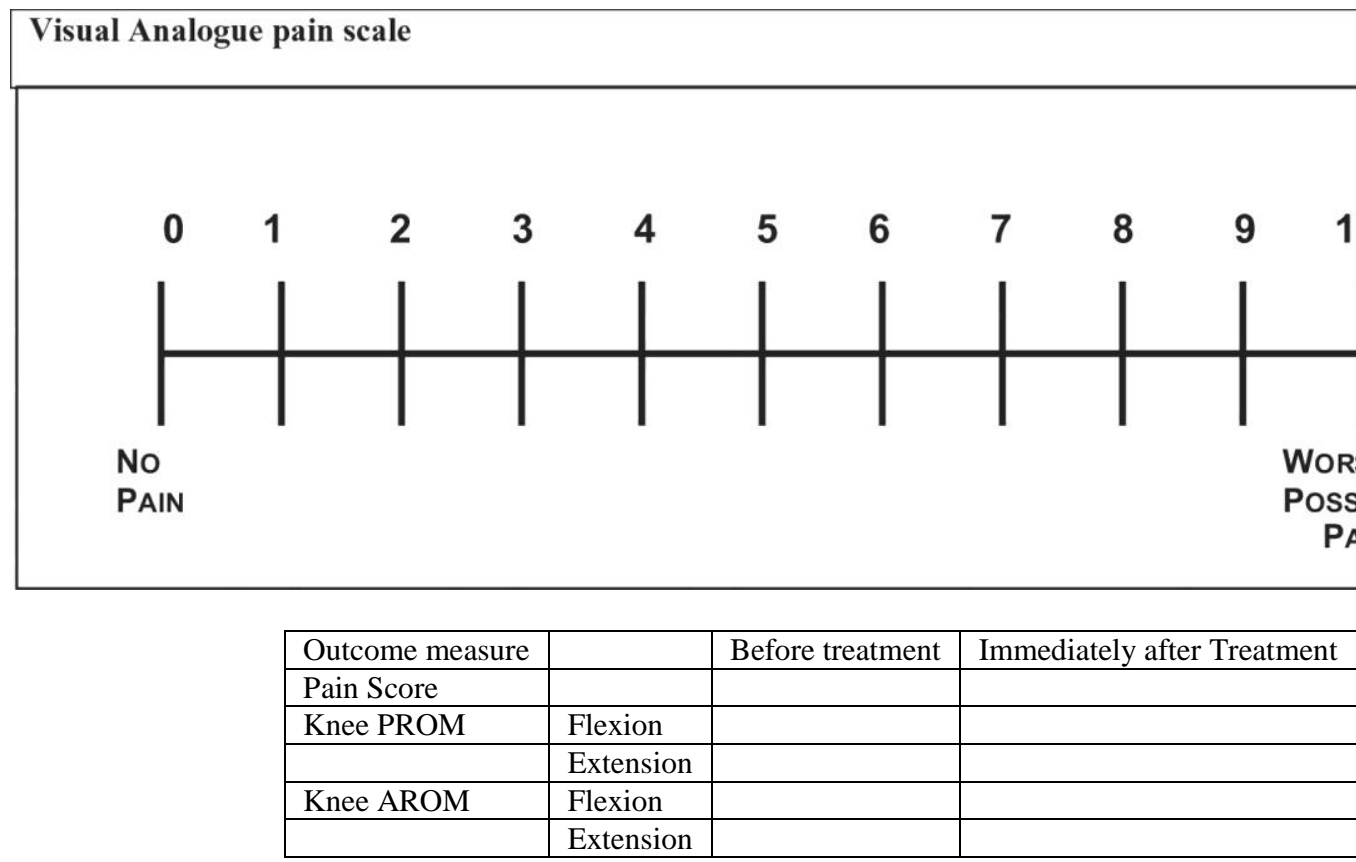

\section{Functional Index Questionnaire}

Patient completes items 1-8 in the appropriate column 0 for the left or right knee

Today, do you or would you have any problem in your knee at all?

1. Walking as far as a mile

$\square$ Unable to do $\square$ Can do with problem $\quad \square$ No Problem

2. Climbing up 2 flights of stairs (16 steps)

3. Squatting

$\square$ Unable to do $\square$ Can do with problem $\quad \square$ No Problem

4. Kneeling

$\square$ Unable to do $\square$ Can do with problem $\quad \square$ No Problem

$\square$ Unable to do $\square$ Can do with problem $\square$ No Problem

5. Sitting for prolonged periods with your knees bent in one position

$\square$ Unable to do $\quad \square$ Can do with problem $\square$ No Problem

6. Climbing up 4 flights of stairs (32 steps)

$\square$ Unable to do $\square$ Can do with problem $\quad \square$ No Problem

7. Running a short distance, say 100 meters. (about the length of a football field)

$\square$ Unable to do $\square$ Can do with problem $\square$ No Problem

8. Walking a short distance (about a city block)

$\square$ Unable to do $\square$ Can do with problem $\square$ No Problem

Volume 5 Issue 7, July 2016 www.ijsr.net

Licensed Under Creative Commons Attribution CC BY 